\title{
Interfacial Ions Sieving for Ultrafast and Complete Desalination through 2D Nanochannel Defined Graphene Composite Membranes
}

Dian Gong, ${ }^{\dagger}, \ddagger, \mathbb{I}$ Yichen Yin, ${ }^{\dagger}, \ddagger$, Il Huiling Chen, ${ }^{\dagger}, \ddagger, \mathbb{I}$ Bing Guo, Ping Wu, ${ }^{\dagger}, \ddagger$ Yue Wang, ${ }^{\dagger}, \ddagger$ Yang

Yang, ${ }^{\dagger}$ Zhikao Li, ${ }^{\S}$ Yue He, ${ }^{*}$, and Gaofeng Zeng*†,

$\dagger$ CAS Key Laboratory of Low-carbon Conversion Science and Engineering, Shanghai Advanced

Research Institute, Chinese Academy of Sciences, Shanghai 201210, China.

$¥$ School of Chemical Engineering, University of Chinese Academy of Science, Beijing 100049, China

" Department of Oral and Maxillofacial-head and Neck Oncology, Shanghai Ninth People's Hospital,

Shanghai Jiao Tong University, Shanghai 200011, China

$\S$ Department of Chemical Engineering, Monash University, Clayton VIC 3800, Australia 


\section{Experimental details}

\section{Materials}

Graphene powder (flake size $0.5-3 \mu \mathrm{m}$ with thickness of $0.8-1.2 \mathrm{~nm}$ ), produced by chemical method, was supplied by Xianfeng Co. (China) and used as received without further treatment. Asymmetric porous alumina tubes supplied by Inopor (Germany) were used as support. The inner and outer diameters and the length of the tubes were 7, 10 and $65 \mathrm{~mm}$, respectively, and the nominal pore size of the inner surface is $\sim 100 \mathrm{~nm}$. Both ends of the support tube were glazed at $900{ }^{\circ} \mathrm{C}$ (Duncan, Fresno, CA) but left with a $\sim 3.0 \mathrm{~cm}$ middle section corresponding to $6.5 \mathrm{~cm}^{2}$ surface area for membrane deposition. The supports were cleaned sequentially by ethanol, 4\% aqueous $\mathrm{KOH}$ solution and hot deionized water before membrane synthesis.

$1 \mathrm{H}, 1 \mathrm{H}, 2 \mathrm{H}, 2 \mathrm{H}-$ perfluorodecanethiol (PFDT, >97\%) and N,N-Dimethylformamide (DMF, >99.5\%) were supplied by Sigmaaldrich. 2,2-azobis-2-methylpropionitrile (AIBN, 98\%), 3-mercaptopropyltrimethoxysilane $(97 \%)$, toluene $(>98.5 \%)$, ethanol $(99.7 \%), \mathrm{NaCl}(>99.5 \%), \mathrm{Na}_{2} \mathrm{SO}_{4}(>99.0 \%)$, $\mathrm{MgSO}_{4} \cdot 7 \mathrm{H}_{2} \mathrm{O}(>99.0 \%)$ and $\mathrm{MgCl}_{2}(>99.0 \%)$ were purchased from Macklin and used as received without further purification.

\section{Preparation of fGraphene membranes / samples}

The fGraphene laminates are firstly prepared through the thiol-ene click reaction between the conjugated $\pi$-bonds of graphene and the thiol groups of $1 \mathrm{H}, 1 \mathrm{H}, 2 \mathrm{H}, 2 \mathrm{H}$-perfluorodecanethiol (PFDT) by using 2,2-azobis-2-methylpropionitrile (AIBN) as a catalyst in a nitrogen purging three-neck flask. In typical, $35 \mathrm{~mL}$ DMF dispersion containing $50 \mathrm{mg}$ graphene was prepared under the help of ultrasonic homogenizer (Qsonica Sonicators Q125, $50 \mathrm{~W}$ ) for $30 \mathrm{~min}$ at room temperature and then was transferred to a $50 \mathrm{~mL}$ three-neck flask, followed by degassing in vacuum and $\mathrm{N}_{2}$ purging for 30 min 
each. Then $150 \mathrm{mg}$ PFDT and $20 \mathrm{mg}$ AIBN were added into the flask. The mixture was stirred at 1500 $\mathrm{rpm}$ and heated to $80{ }^{\circ} \mathrm{C}$ and then kept $12 \mathrm{~h}$ with reflux condensation under the protection of $\mathrm{N}_{2}$ flow (Figure S26). The centrifugation collected products were thoroughly cleaned with DMF at $70{ }^{\circ} \mathrm{C}(4$ times* $1 \mathrm{~h}$ per time) and ethanol at $40{ }^{\circ} \mathrm{C}(3$ times $* 40$ min per time $)$ in sequence by repeating centrifugation / ultrasonic re-dispersion. To select small size of fGraphene monolayer, the washed fGraphene was re-dispersed in DMF and then centrifugalized with $800 \mathrm{rpm}$ for $5 \mathrm{~min}$. The fGraphene dispersed in the supernatant was collected and then centrifugalized at $10000 \mathrm{rpm}$ to fGraphene powder. Then fGraphene was dried at $70{ }^{\circ} \mathrm{C}$ for $12 \mathrm{~h}$ and freeze-dried for $6 \mathrm{~h}$.

The porous ceramic tube was grafted with thiol groups through treating it in 3-mercaptopropyltrimethoxy-silane toluene solution. The ceramic tube was wrapped by Taflon tape to protect the unwanted outer surface. In typical, $0.22 \mathrm{~g}$ 3-mercaptopropyl- trimethoxy-silane was dissovled in 100 $\mathrm{mL}$ toluene. The support tube was immersed in the solution and refluxed under $\mathrm{N}_{2}$ at $110{ }^{\circ} \mathrm{C}$ for $1 \mathrm{~h}$. The activated support was then rinsed with ethnaol to remove residual reagent and solvent. The activated support was put into the three-neck flask, which contains graphene DMF suspension (1 mg graphene per $\mathrm{mL}$ DMF, $30 \mathrm{~mL}$ ). Then $20 \mathrm{mg}$ AIBN was added into the flask. The mixture was stirred at $1500 \mathrm{rpm}$ and heated to $80^{\circ} \mathrm{C}$ and then kept for $12 \mathrm{~h}$ with reflux condensation under the protection of $\mathrm{N}_{2}$ flow (Figure S16). After reaction, the support was rinsed with ethanol and dried at room temperature.

The supported fGraphene membranes were prepared by pressure filtration. Ceramic support was mounted into the tube housing and sealed with two O-rings on the both glazed ends of support. The upper end of tube housing was closed by stop-valve and the bottom end was connected to the container of fGraphene ethanol suspension. The fGraphene suspension was drove to the lumen side of tube through the bottom end by pressurized nitrogen. Liquid was collected and measured from the 
permeation side of ceramic tube. In a typical synthesis, $2 \mathrm{mg}$ fGraphene powder was dispered in $250 \mathrm{~mL}$ ethanol by an ultrasonic homogenizer for $30 \mathrm{~min}$. Then the suspension was transferred to the synthesis container and drove by an increasing $\mathrm{N}_{2}$ pressure from 1 to 8 bar. The as-prepared composite membrane was dried overnight at $40{ }^{\circ} \mathrm{C}$ in vacuum. On the other hand, the reference $\mathrm{GO}$ and graphene membranes were prepared by using the similar method. The GO membrane was prepared by filtrating $250 \mathrm{~mL}$ of GO aqueous $\left(0.002 \mathrm{mg} \mathrm{GO} \mathrm{mL}^{-1}\right)$ under 2-12 bar $\mathrm{N}_{2}$. The graphene membrane was obtained by the same method to fGraphene (250 $\mathrm{mL}$ ethanol suspension contain $2 \mathrm{mg}$ graphene).

\section{Desalination}

The desalination performance of membranes was mainly studied by the direct-contact vacuum membrane distillation (VMD) method at 40-80 ${ }^{\circ} \mathrm{C}$. As the reference sample GO membrane has the capacity to "dissolve" water, the same separation method could be called as pervaporation for GO membrane. The membrane was fixed with O-rings in the separator. Then the separator was vertically fixed in an oven. The saline water was fed to the lumen side of the membrane with a flow rate of $2 \mathrm{~mL}$ $\min ^{-1}$ by a constant flow pump. The effluent of the lumen side was returned to the feedstock tank. The retentate side of the separator was connected in sequence to a sample collector, which was immersed in a liquid nitrogen cold trap, and a vacuum pump, by which the pressure of retentate side was kept around $100 \mathrm{~Pa}$. Collection of the permeated liquid was begun after $30 \mathrm{~min}$ running of the system under the setting pressure and temperature. The volume of the permeated liquid was measured by volumetric cylinders. The collection interval, which depended on the permeability of membrane, varied between 1 to $3 \mathrm{~h}$. The concentrations of sodium salts were determined by conductivity using a conductivity meter (MP523-03, Sanxin) equipped a type 7801 sodium ion electrode and confirmed by an inductively 
coupled plasma atomic emission spectrometry (ICP-AES, PerkinElmer Optima 8000). The concentrations of magnesium salts were measured by ICP-AES.

The membrane performance was evaluated by the ion rejection $(\mathrm{R})$ and water flux $\left(\mathrm{F}, \mathrm{L} \mathrm{m}^{-2} \mathrm{~h}^{-1}\right)$ determined as equations (1) and (2):

$$
\begin{gathered}
\mathrm{R}=\left(1-C_{i} / C_{0}\right) * 100 \% \\
F=V /(A * t)
\end{gathered}
$$

where $C_{0}$ and $C_{i}\left(\mathrm{~mol} \mathrm{~L}^{-1}\right)$ are the salt concentrations of the feed solution and permeate liquid, respectively, $V$ is the volume of the permeate liquid (L), $A$ is the membrane area $\left(\mathrm{m}^{2}\right)$, and $t$ is the permeation time $(\mathrm{h})$.

The liquid entry pressure (LEP) for fGraphene membranes were measured by using the setup similar to reverse osmosis (Figure S12). ${ }^{1} 100 \mathrm{~mL}$ pure water passes through the shell side of membrane module and 1 wt.\% $\mathrm{NaCl}$ solution was fed into the membrane side under the controllable pressure. The conductivity of the recycled pure water was monitored by a conductivity electrode. With the slightly increasing of feeding pressure, LEP was equal to the feed pressure that a sharp increase of conductivity was recorded in the pure water recycling tank.

The desalination on fGraphene membrane was tested by a temperature controllable reverse osmosis (RO) method (Figure S20). Limited by the LEP of fGraphene membrane, RO was carried out by using a relatively low concentration $\mathrm{NaCl}$ solution (2000 ppm) with a feeding pressure of 3 bar at room temperature and the elevated temperature. The water flux was determined by the weight of collected liquid (eq. 2) and the rejection was calculated by the conductivity of collection (eq. 1). The specific water flux $\left(S_{F}\right)$ was calculated by the equation (3):

$S_{F}=F /\left(P_{N 2}-P_{O}\right)$ 
where $F$ is the water flux $\left(\mathrm{L} \mathrm{m}^{-2} \mathrm{~h}^{-1}\right), P_{N 2}$ is the $\mathrm{N}_{2}$ driven pressure of feed and $P_{O}$ is the osmosis pressure of feed solution.

The water flux over fGraphene membrane was tested via the forward osmosis method at room temperature and $80{ }^{\circ} \mathrm{C}$ (Figure $\left.\mathrm{S} 21\right) . \mathrm{NaCl}$ solutions with different concentrations were introduced into the shell side of membrane module as the draw solution and then returned to the feed tank. Pure water or 3 wt.\% $\mathrm{NaCl}$ solution was introduced into the lumen side of membrane tube. The water flux was determined by the volume change of draw solution during the testing time.

\section{Characterizations}

The morphology and thickness of membranes were characterized by a field emission scanning electron microscope (FESEM, Zeiss SUPRA 55 SAPPHIRE) with an acceleration voltage of $2 \mathrm{kV}$. The high-resolution structure of fGraphene fragment was measured by a transmission electron microscopy (TEM, JEM-2100F, $200 \mathrm{kV}$ ) and a spherical aberration corrected TEM (ACTEM, JEM-ARM300F, 80 $\mathrm{kV}$ ). The elemental distribution of fGraphene membranes was detected by energy dispersive spectrometers (EDS, Oxford Instrument and JED-2300 Analysis Station) equipped on SEM and ACTEM, respectively. The thickness of fGraphene and graphene monolayer was characterized by atomic force microscope (AFM, Bruker Dimension Icon). The structure of fGraphene membranes was determined by X-ray diffraction (XRD, Rigaku Ultima IV) in the range of 2 theta $5-35^{\circ}$ using $\mathrm{Cu} \mathrm{K} \alpha$ radiation $(\lambda=0.15406 \mathrm{~nm}, 40 \mathrm{kV}, 40 \mathrm{~mA})$.

The surface chemistry of membranes was analyzed by X-ray photoelectron spectroscopy (XPS, Thermo Fisher, K-Alpha) and Fourier transform infrared spectroscopy (FTIR, Nicolet 6700). XPS peak positions were calibrated with the help of the $\mathrm{C} 1 \mathrm{~s}$ peak at $284.8 \mathrm{eV}$. The carbon state of graphene and fGraphene samples was determined by Raman spectroscopy (Thermo Scientific DXR2xi, $\lambda=532 \mathrm{~nm}$ ). The water 
contact angles of membranes were measured by a contact angle tester (Maist Drop Meter A-100P) using a $3 \mu \mathrm{L}$ water droplet. Surface charging behaviors in terms of zeta potential of GO, graphene and fGraphene materials were determined by an electrokinetic analyzer (Malvern ZS90, UK) in the $\mathrm{pH}$ range $3-11$. 
Figures

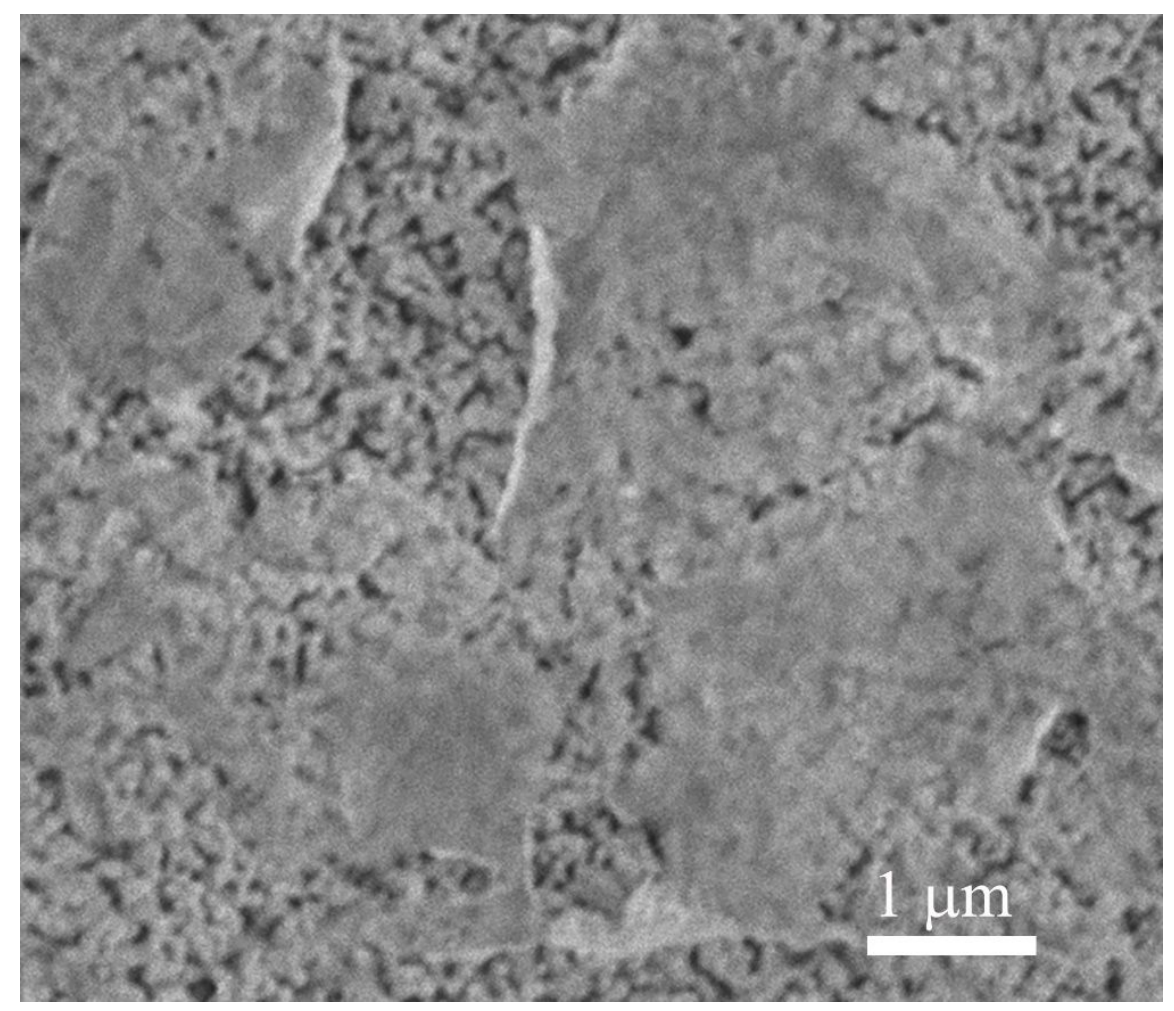

Figure S1 SEM image of support after the interface layer deposition. 


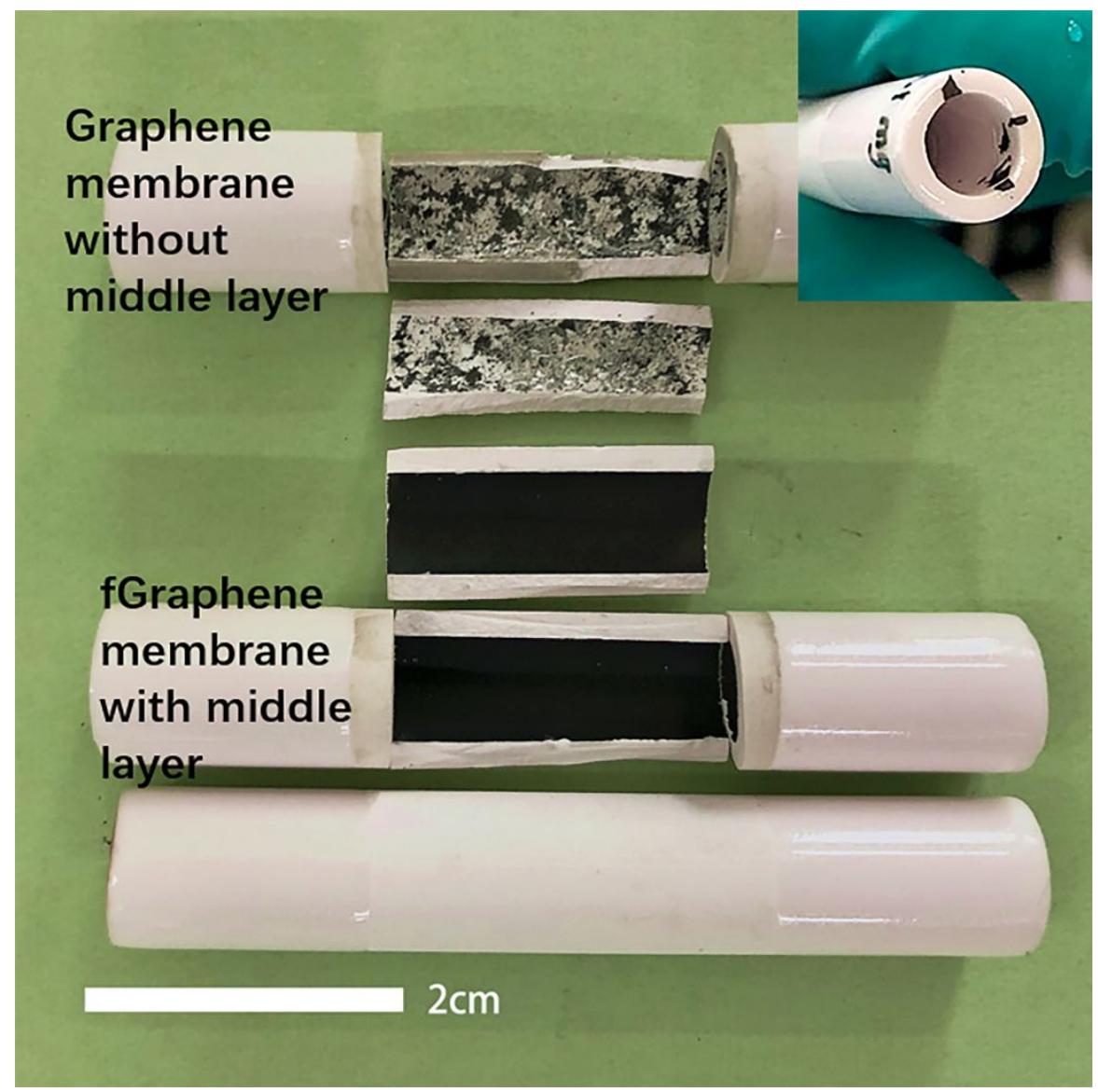

Figure S2 Optical image of graphene based membranes with or without fixed graphene interface layer (Inset: top view of graphene membrane without middle layer treatment). 


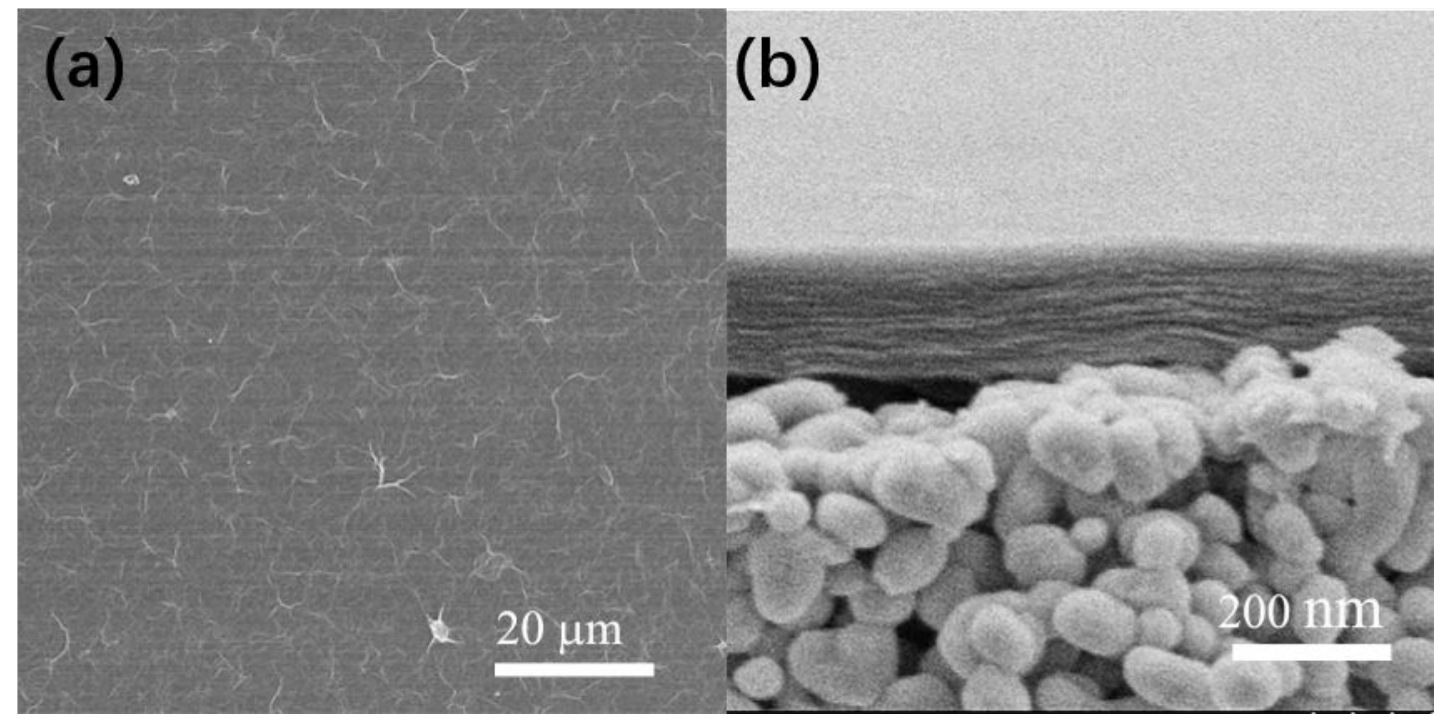

Figure S3 SEM images of (a) surface and (b) cross-section of graphene oxide membrane. 


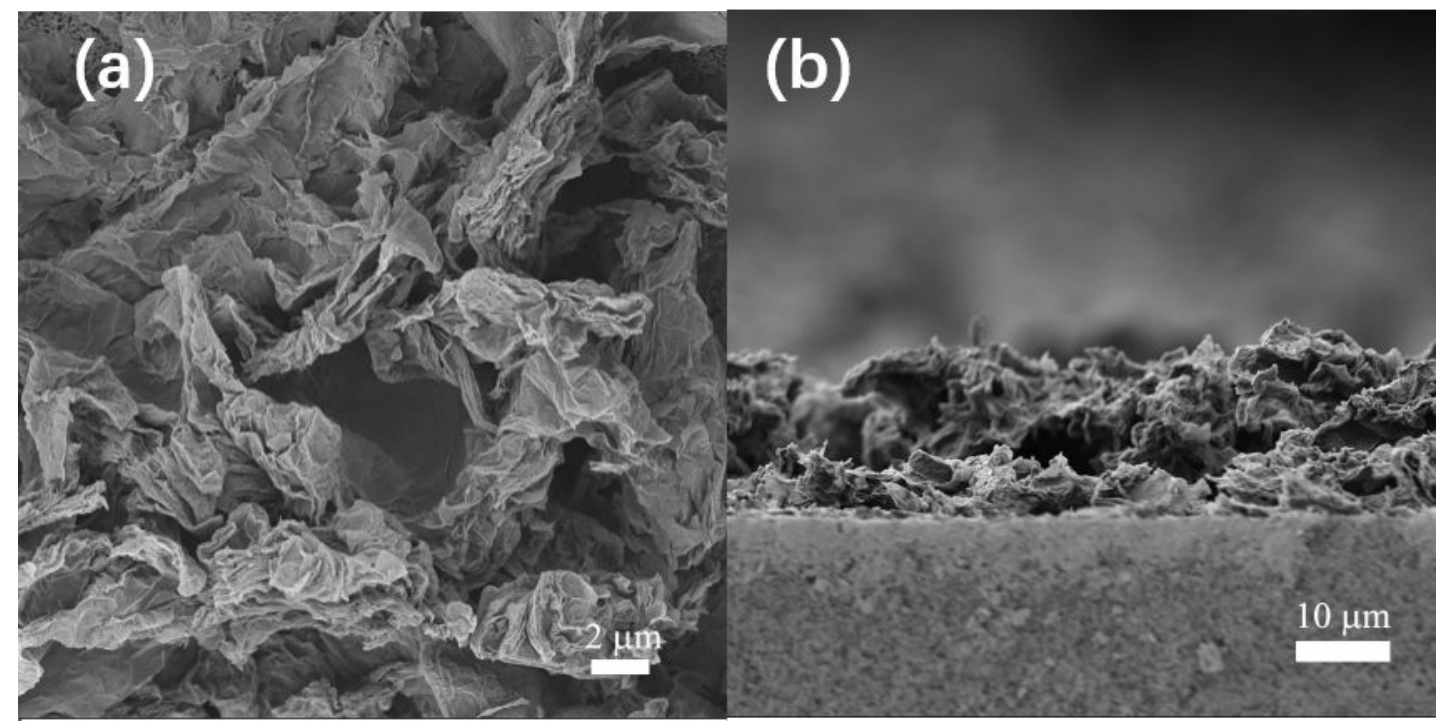

Figure S4 SEM images of (a) surface and (b) cross-section of graphene membrane. 


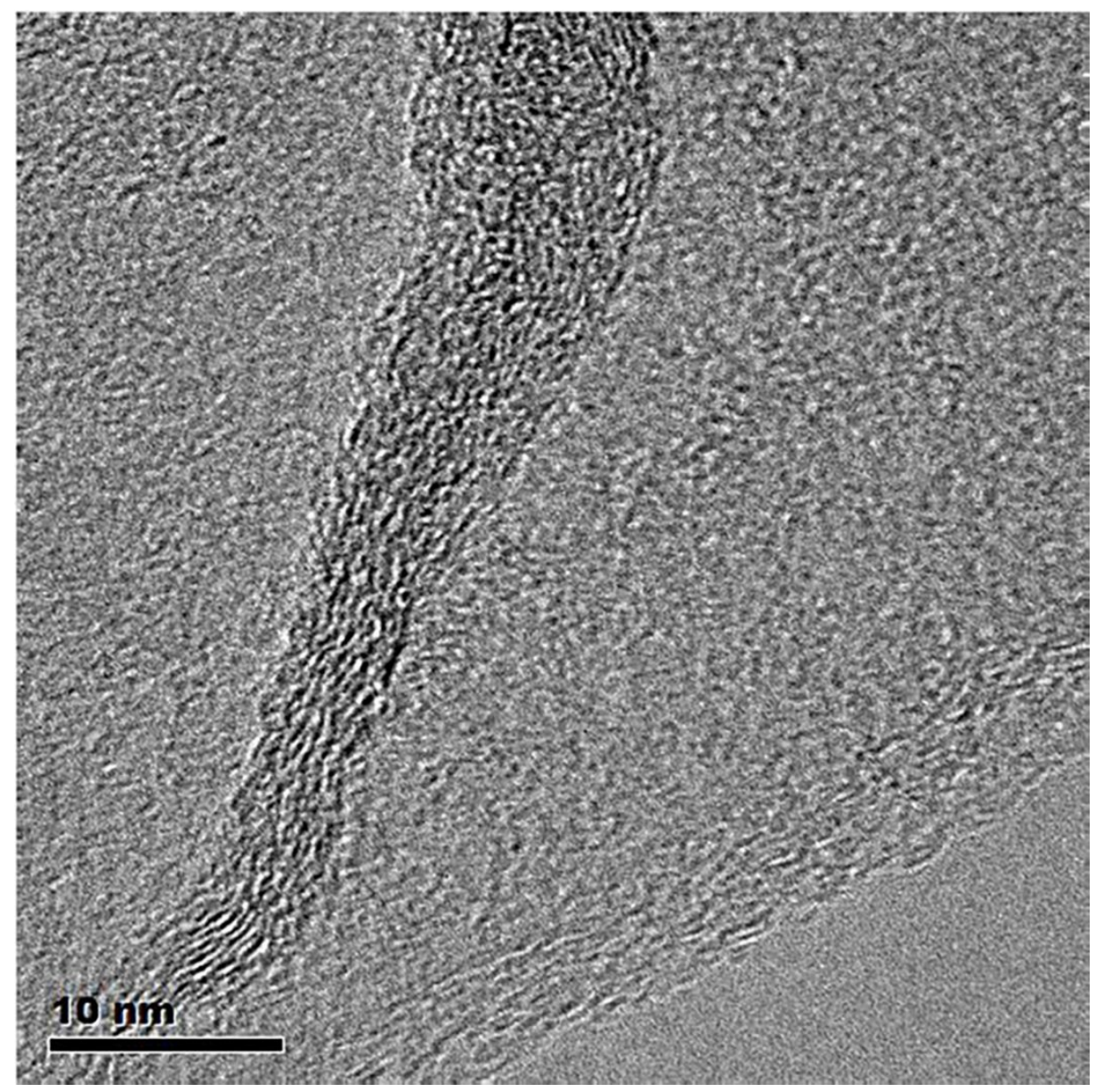

Figure S5 HRTEM image of graphene fragment. 

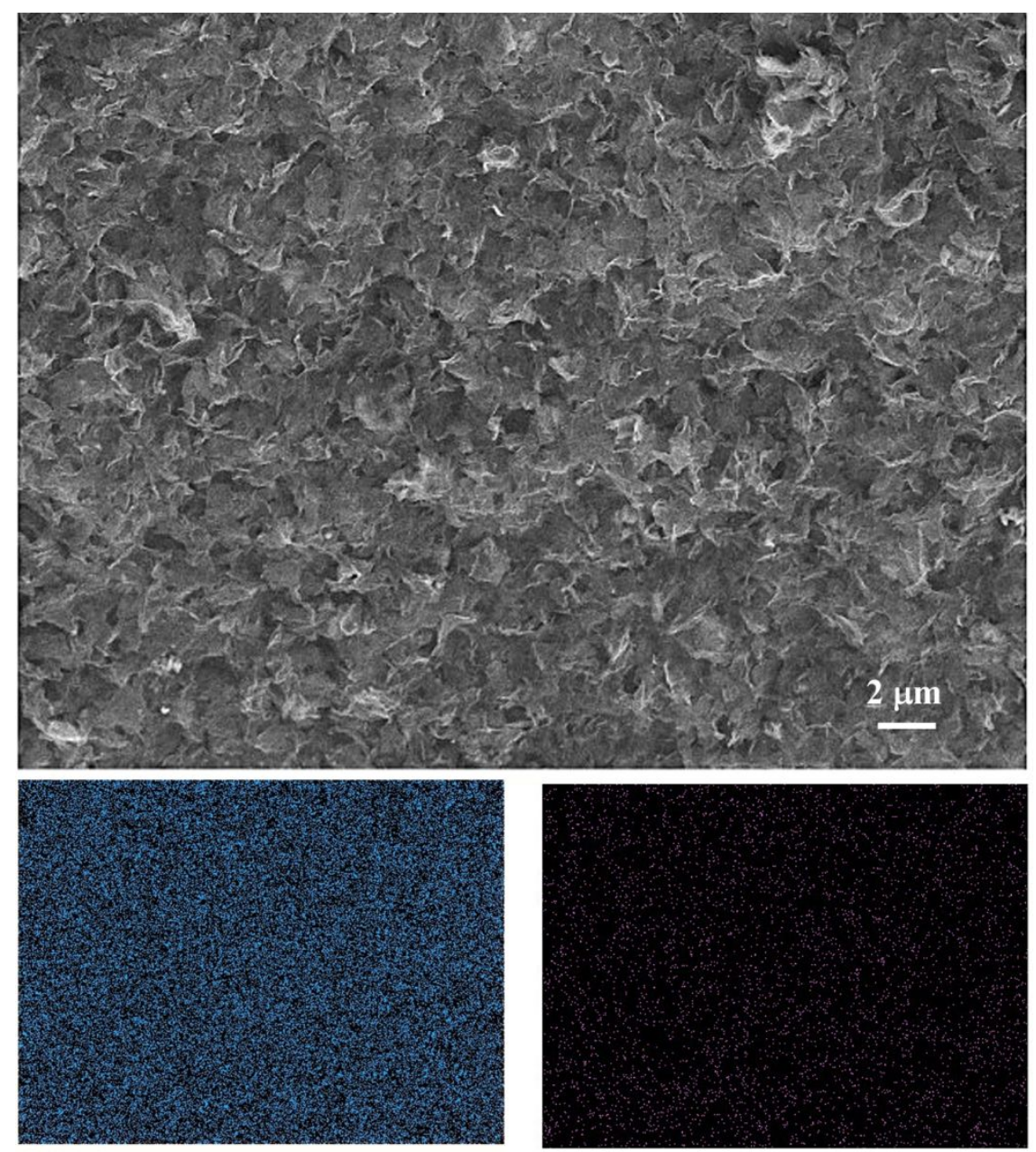

CKa1_2
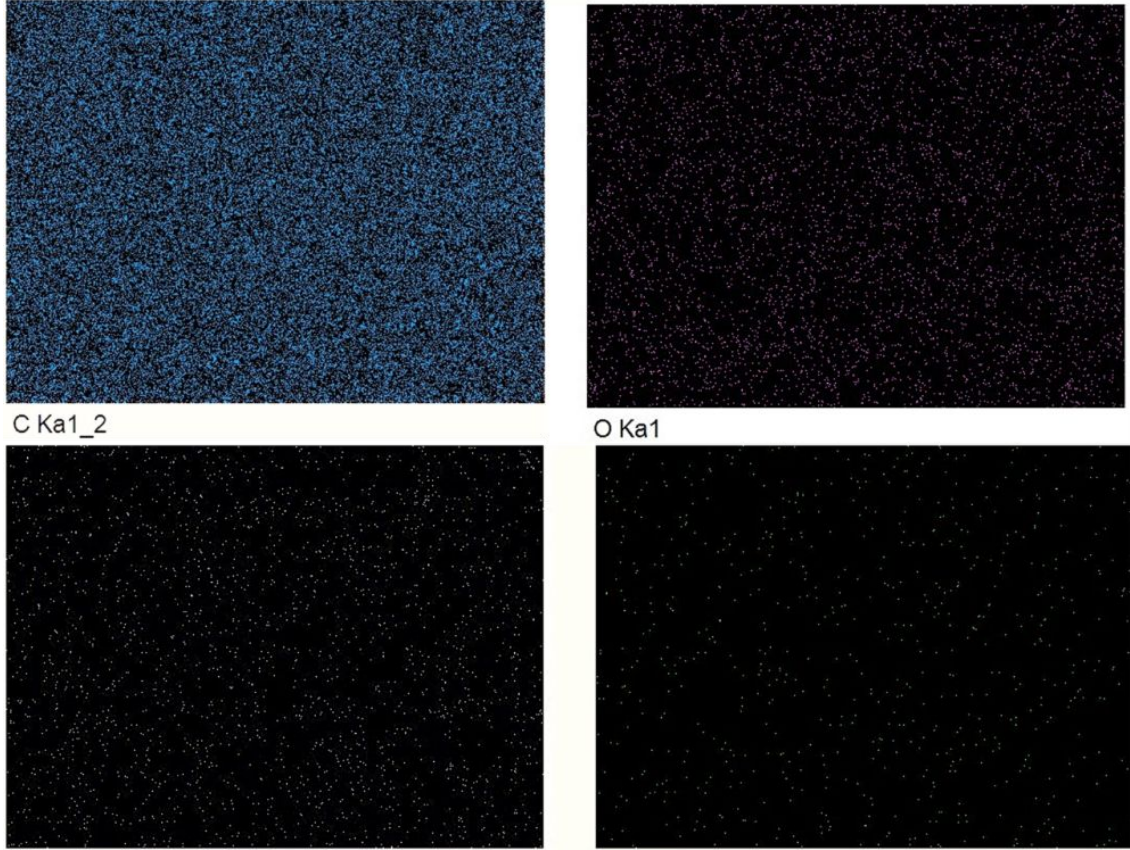

O Ka1

F Ka1_2

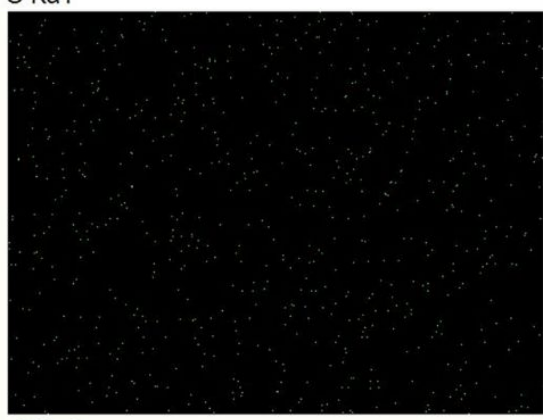

S Ka1

Figure S6 Elemental distribution on fGraphene membrane surface. 
(a)
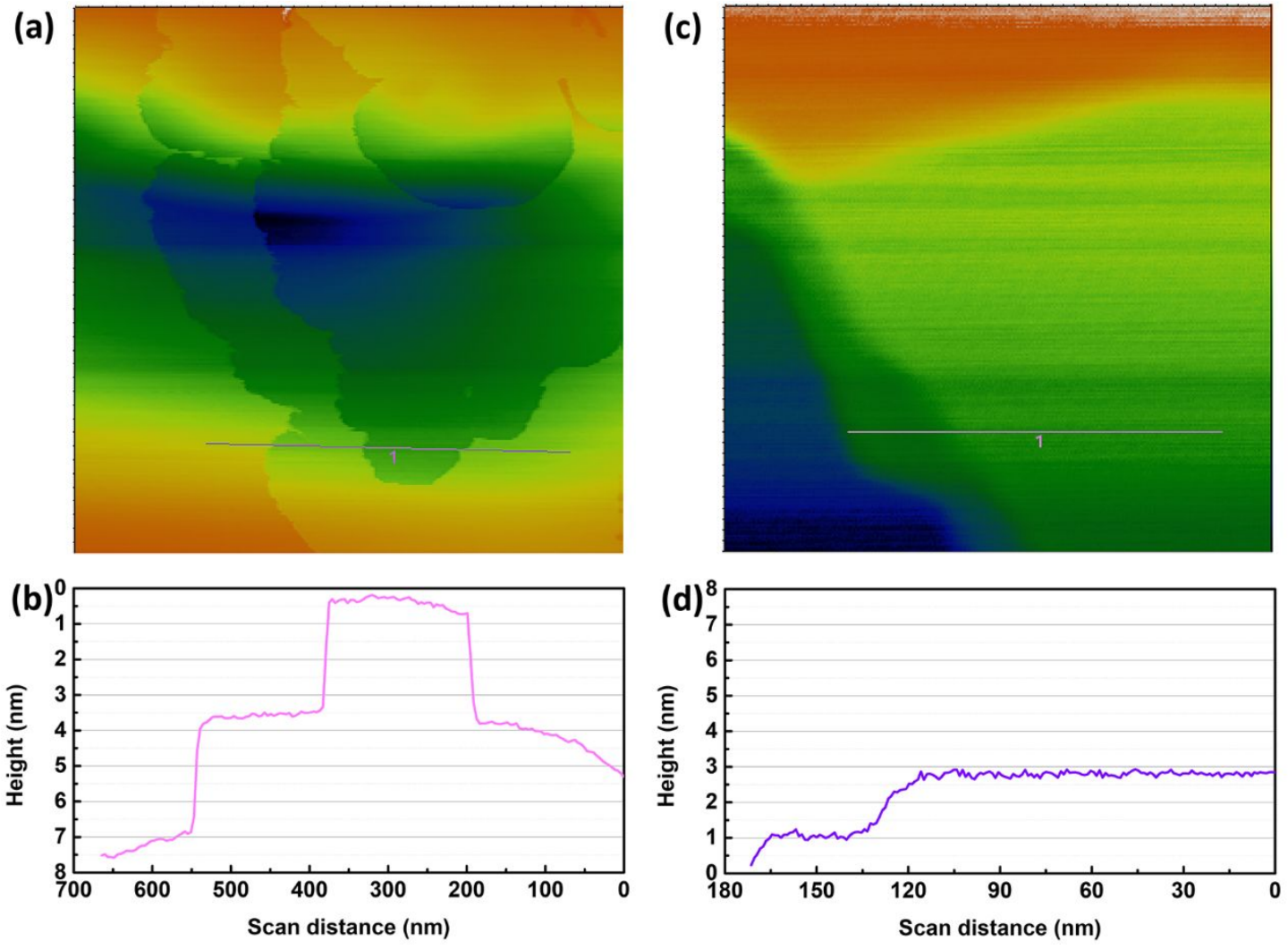

Figure S7 Atomic force microscopy (AFM) measurements of fGraphene few layers (a) and graphene few layers (c), and the height profiles of fGraphene (b) and graphene (d). 
(a)

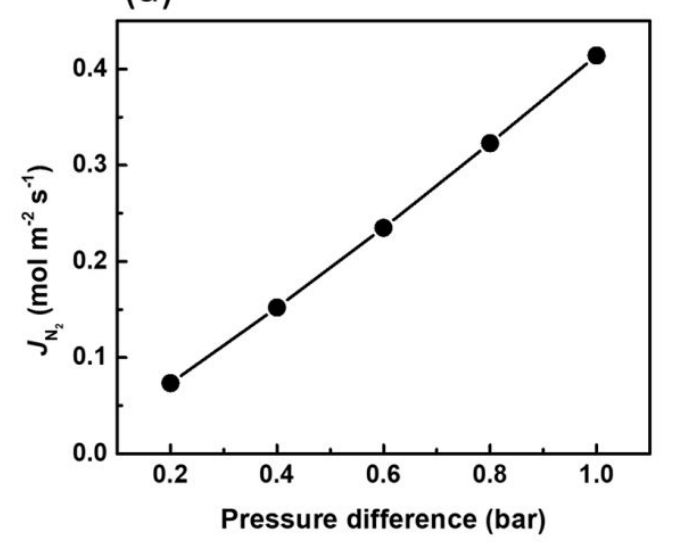

(b)

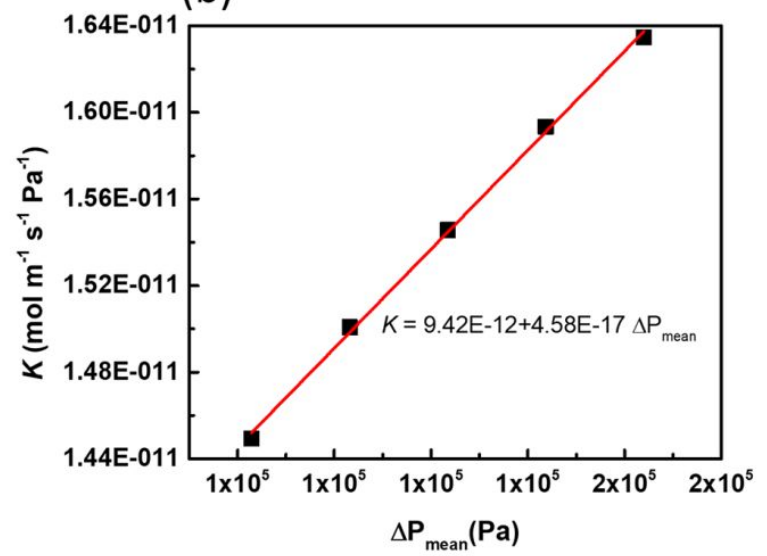

Figure S8 (a) Pressure dependence of $\mathrm{N}_{2}$ flux on fGraphene membrane and (b) pressure dependence of gas permeability coefficient $K$ over fGraphene membrane.

The mean pore size of fGraphene membrane was estimated by Yasuda-Tsai equation as following:

$d_{p}=\left(\frac{B_{0}}{K_{0}}\right)\left(\frac{16}{3}\right)\left(\frac{2 R T}{M \pi}\right)^{1 / 2}$

where $d_{p}$ is the mean pore size of membrane, $B_{0}$ the geometric factor of the membrane, $K_{0}$ is the Knudsen permeability coefficient, $R$ is the gas content, $T$ is the temperature $(298 \mathrm{~K})$ and $M$ is the molecular weight of gas. $B_{0}$ and $K_{0}$ can be calculated by the equation of gas permeability coefficient $(K)$ :

$K=K_{0}+\frac{B_{0}}{\eta} \Delta \bar{p}$

where $\eta$ is the viscosity of the permeant gas $\left(0.00018 \mathrm{~Pa}\right.$ s for $\left.\mathrm{N}_{2}\right)$ and $\Delta \bar{p}$ is the mean pressure of the gas on both sides of a membrane. The gas flux $(J)$ can be expressed as:

$J=K \Delta P / l$

where $\Delta P$ is the pressure difference across the membrane and $l$ is the membrane thickness $(4 \mu \mathrm{m})$. The $\mathrm{N}_{2}$ fluxes over the fGraphene membrane were measured under the pressure difference $(\Delta P)$ from 0.2 to 1.0 bar at room temperature (Figure S8a). ${ }^{2}$ Then $K_{0}$ and $B_{0}$ can be obtained as $9.42 \times 10^{-12}$ and $8.24 \times 10^{-21}$,respectively, by using Eq. (5) and (6). Therefore, the mean pore size of fGraphene membrane was $34.9 \mathrm{~nm}$ estimated by Eq. (4). 


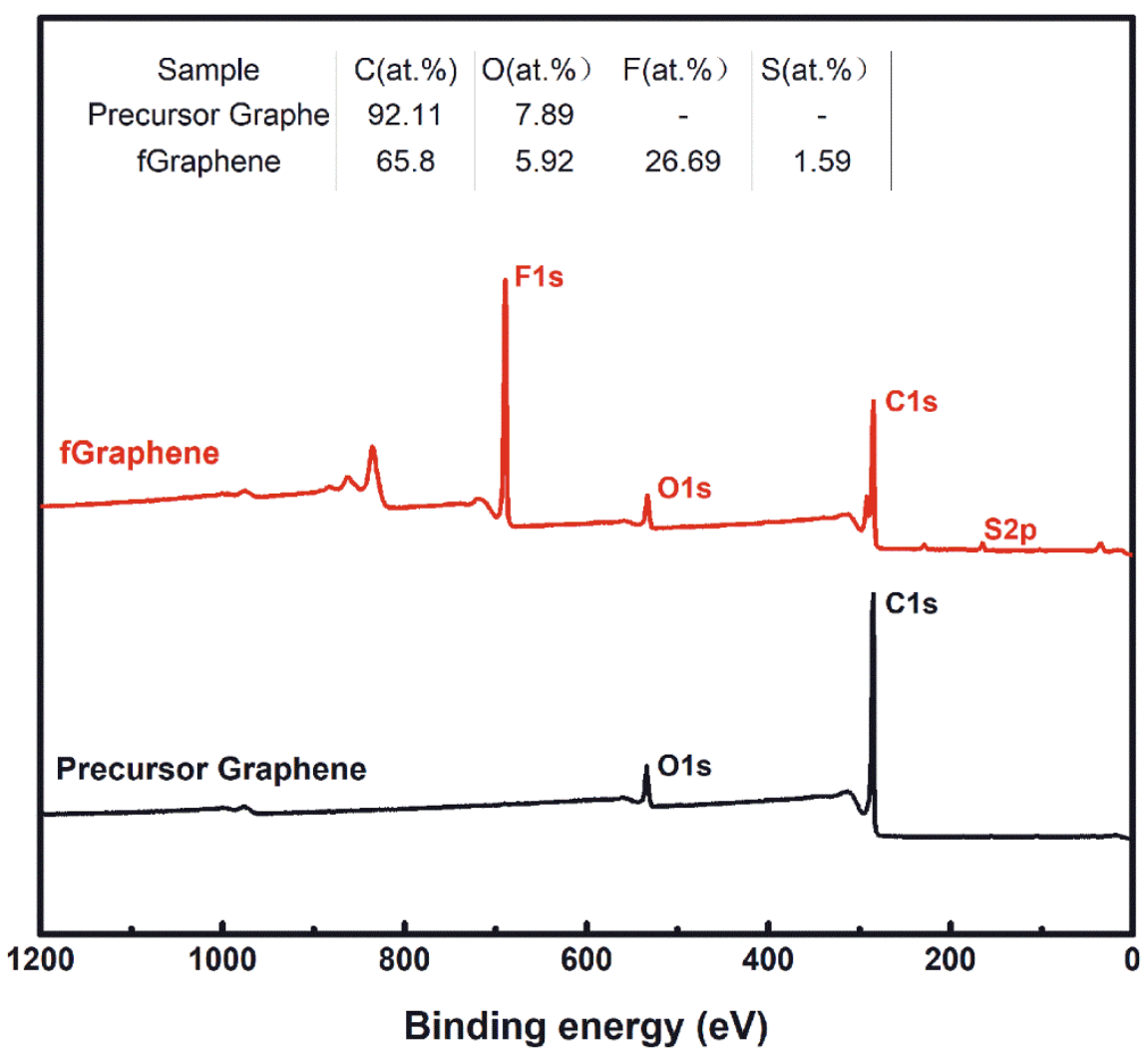

Figure S9 XPS survey of fGraphene and precursor graphene. 


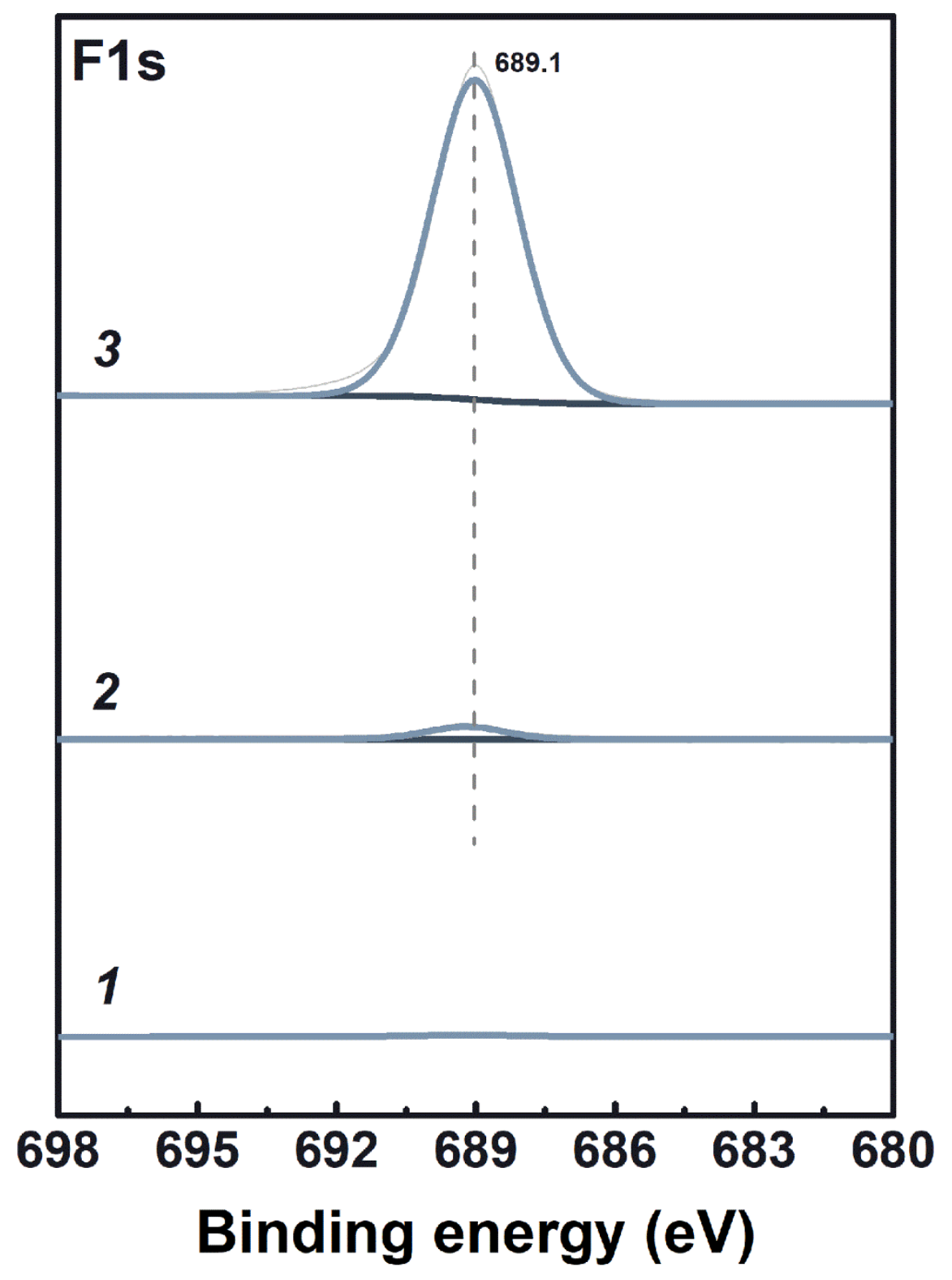

Figure S10 F1s XPS spectra of (1) precursor graphene, (2) graphene sample recovered from the graphene-PFDT physical mixture and (3) fGraphene. 


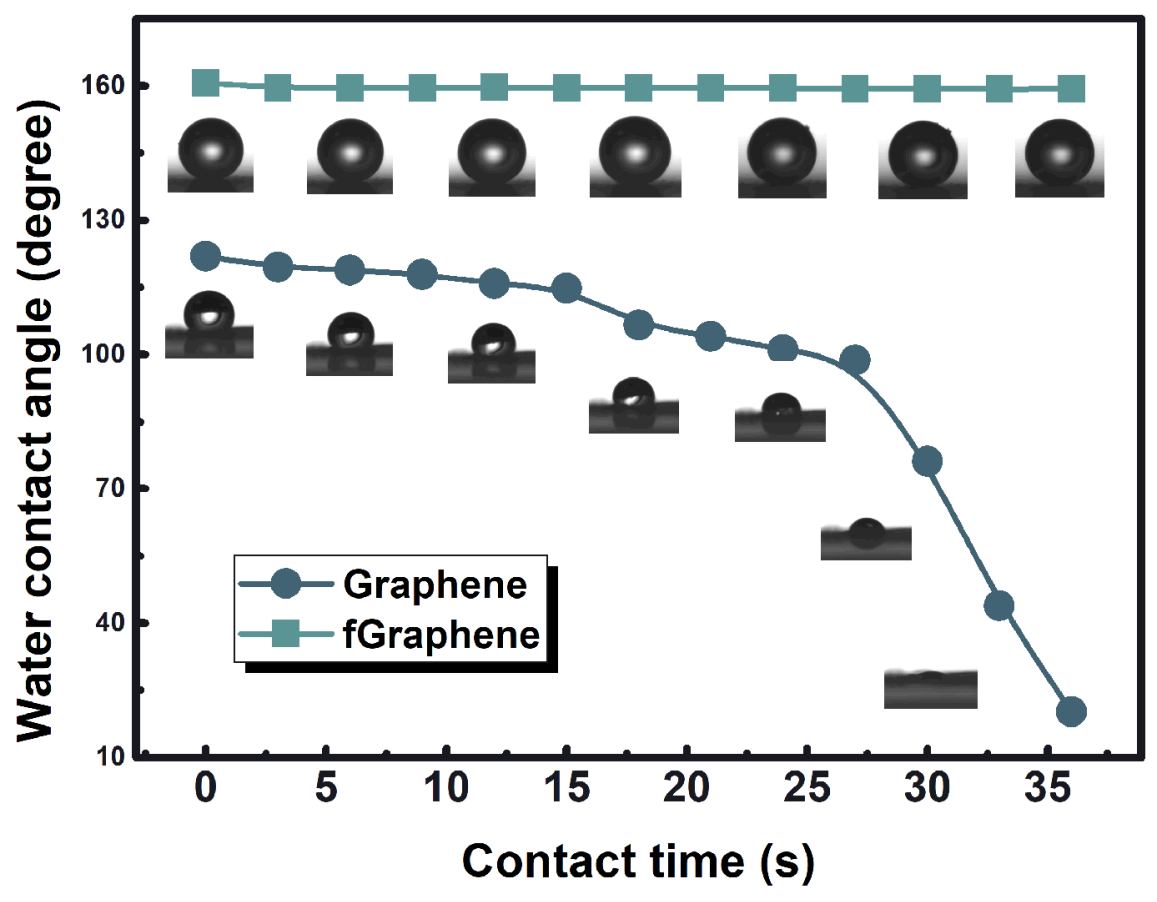

Figure S11 The dynamic water contact angle on fGraphene and graphene membranes. 


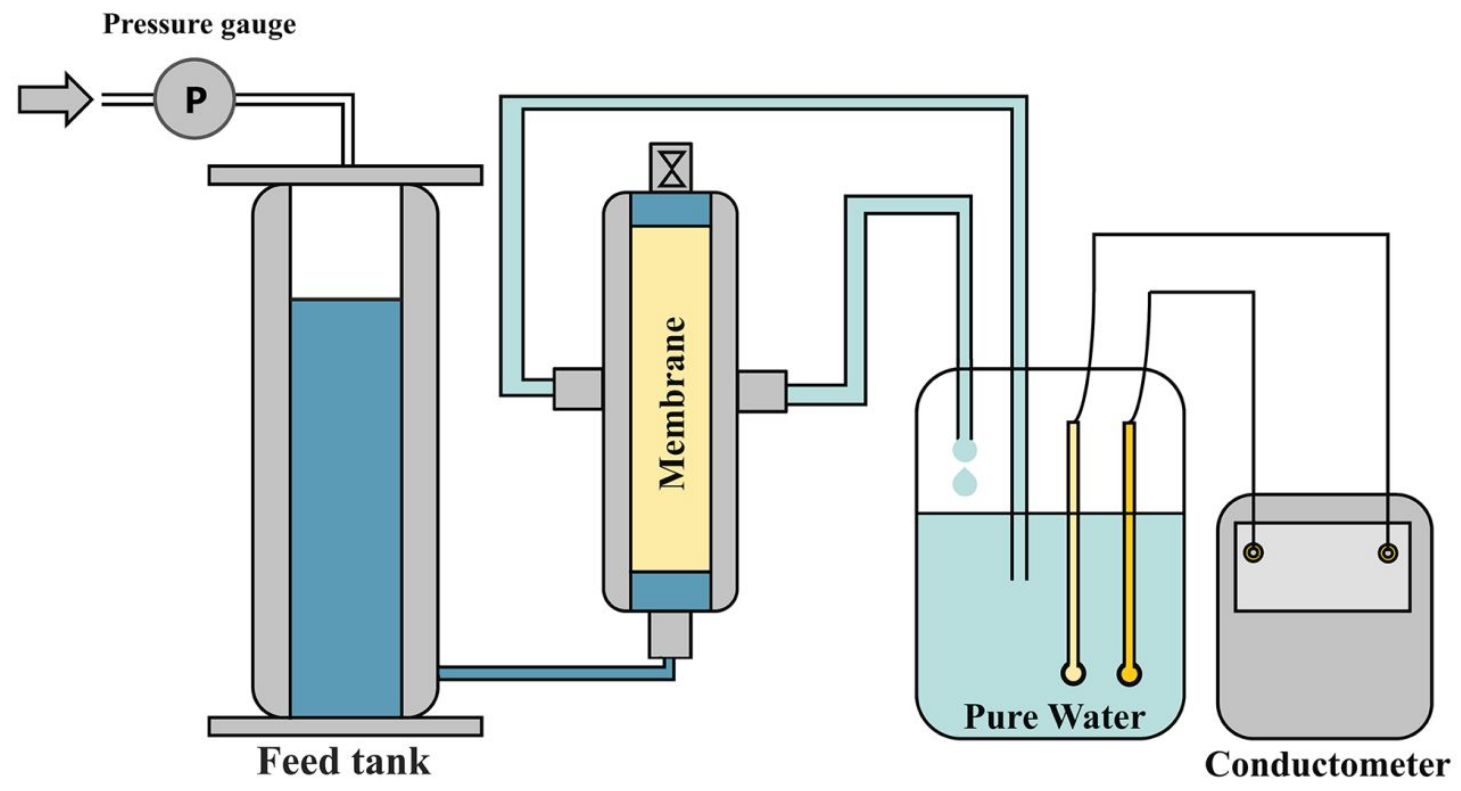

Figure S12 Schematic of the measurement system for liquid entry pressure. 


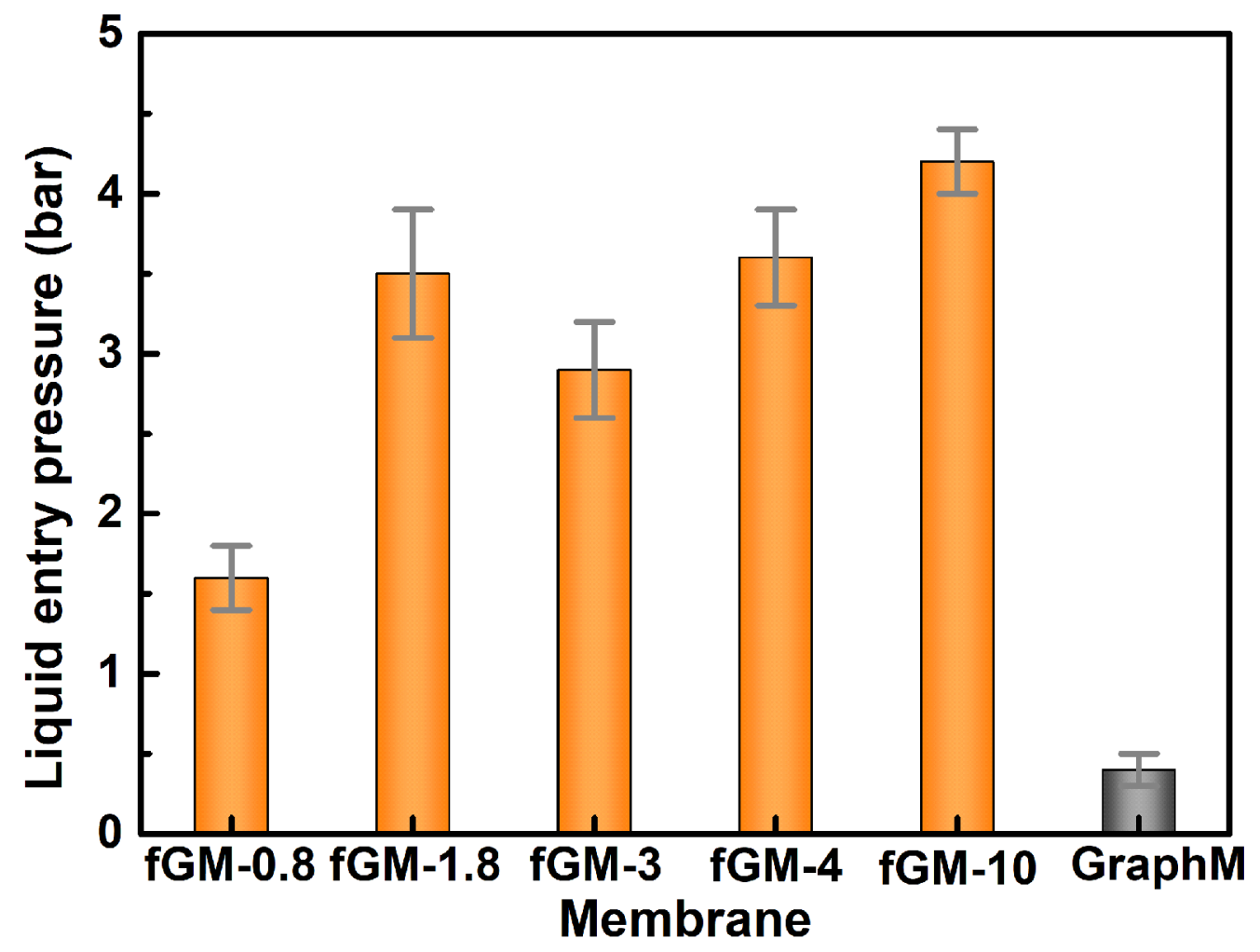

Figure S13 The liquid entry pressure of fGraphene membranes and graphene membrane (fGM- $x$ refers to fGraphene membrane with the approximate thickness of $x \mu \mathrm{m}$ while GraphM means graphene membrane reference). 

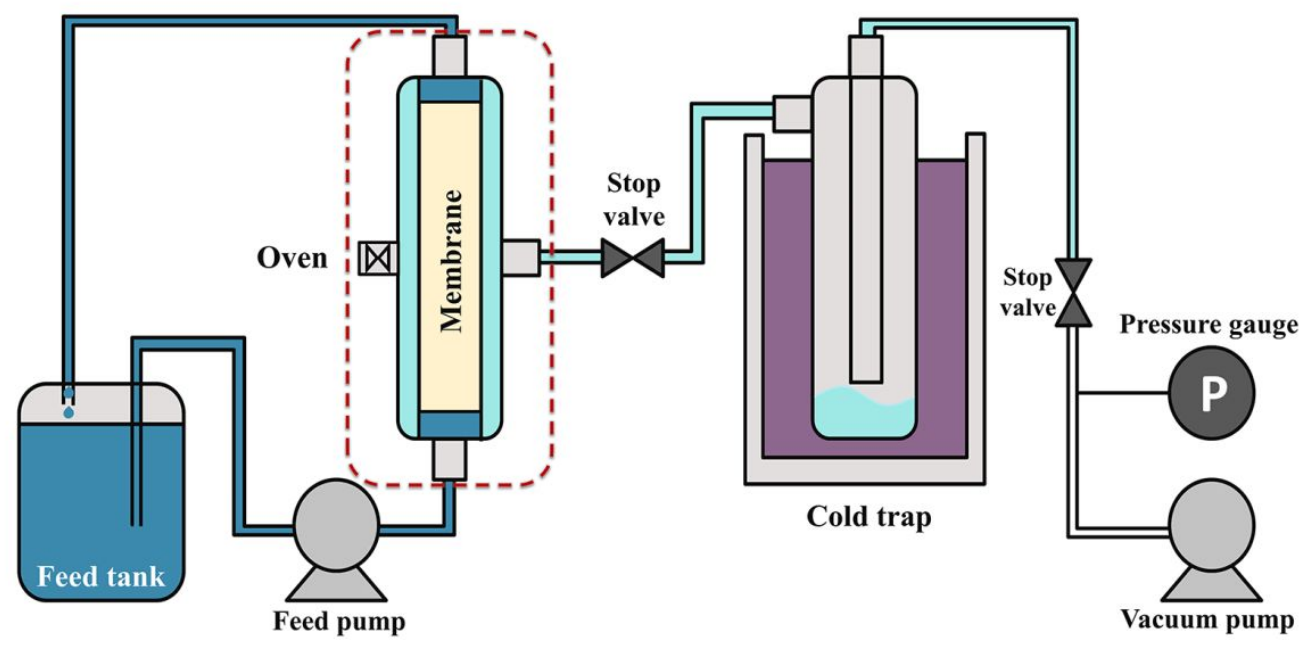

Figure S14 Schematic of the membrane system for vacuum membrane distillation / pervaporation measurements. 
a

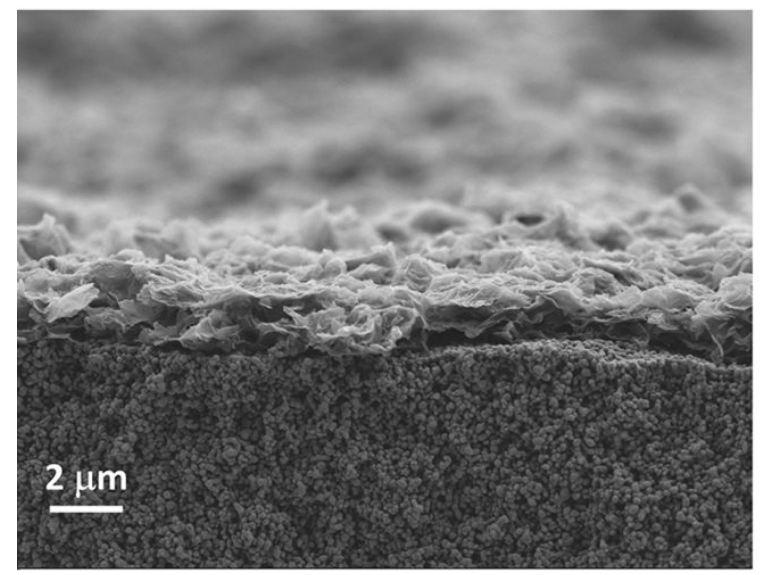

$\mathrm{C}$

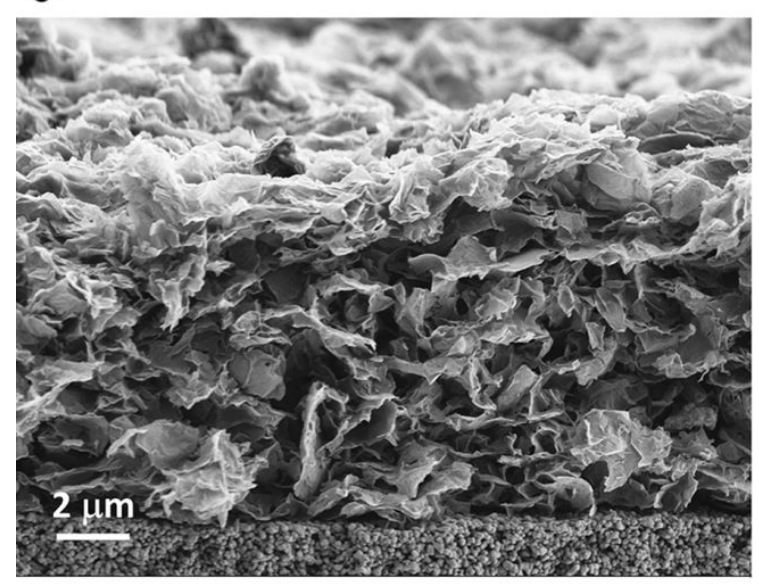

b

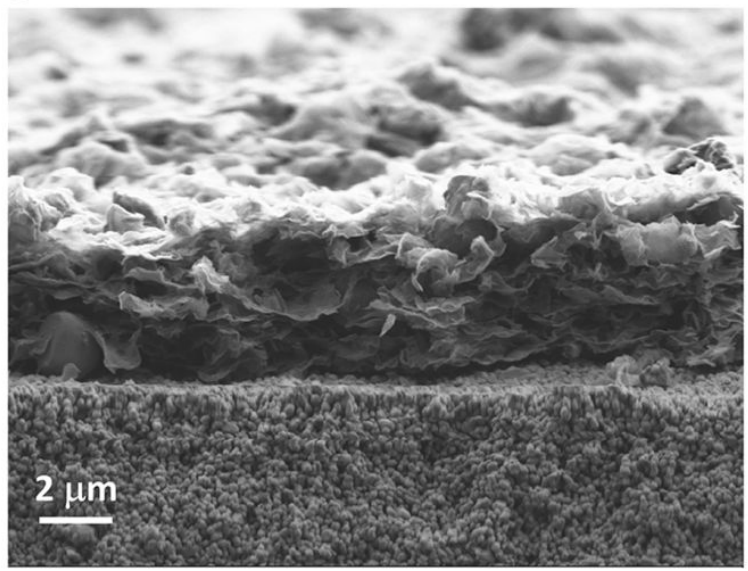

d

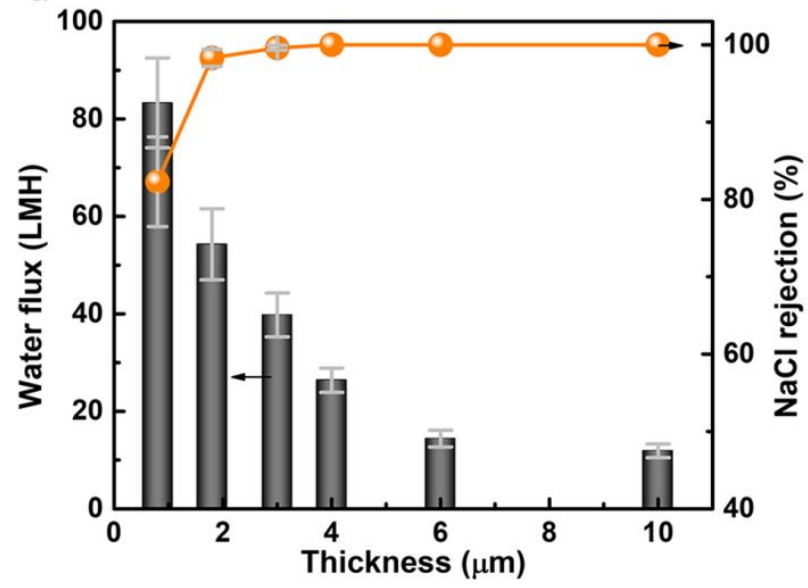

Figure S15 The fGraphene membranes with different thickness, (a) $\sim 1.8 \mu \mathrm{m}$, (b) $\sim 3 \mu \mathrm{m}$, (c) $\sim 6 \mu \mathrm{m}$ and (d) the effects of thickness on the water fluxes and $\mathrm{NaCl}$ rejections ( $1 \mathrm{wt} . \% \mathrm{NaCl}$ solution, $70{ }^{\circ} \mathrm{C}$, VMD). 


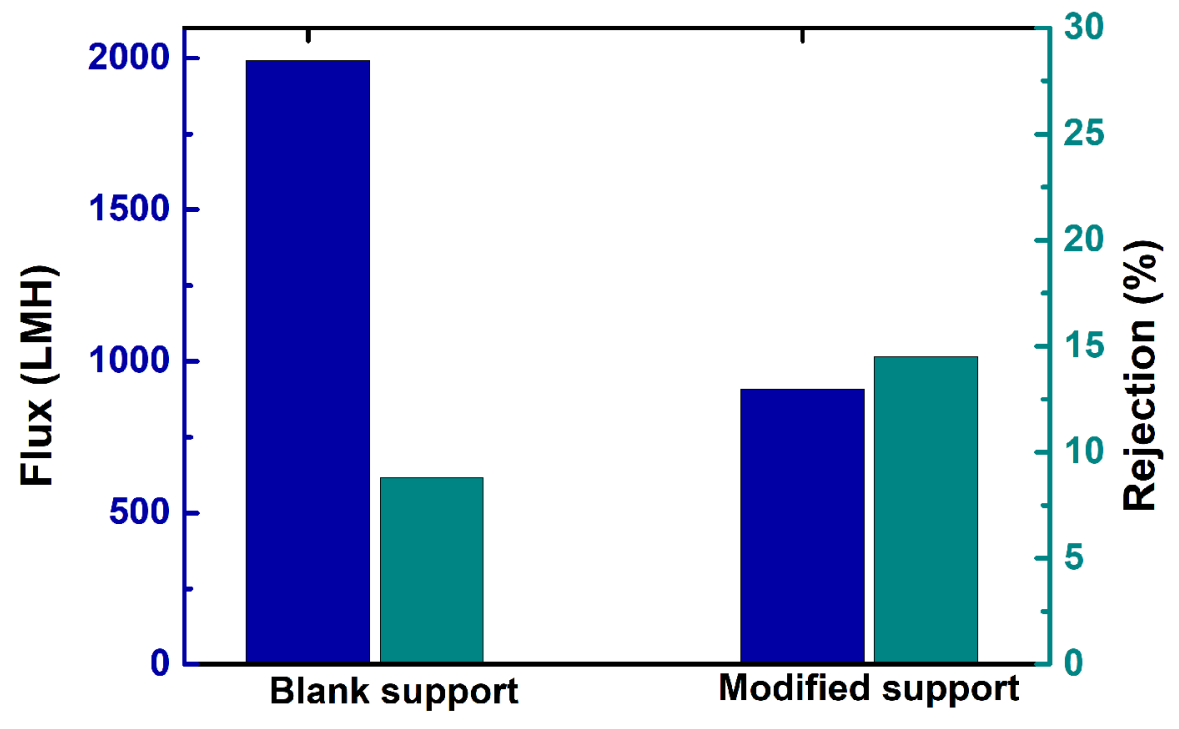

Figure S16 Desaliantion performance of blank support and modified support (1 wt.\% $\left.\mathrm{NaCl}, 70{ }^{\circ} \mathrm{C}\right)$. 


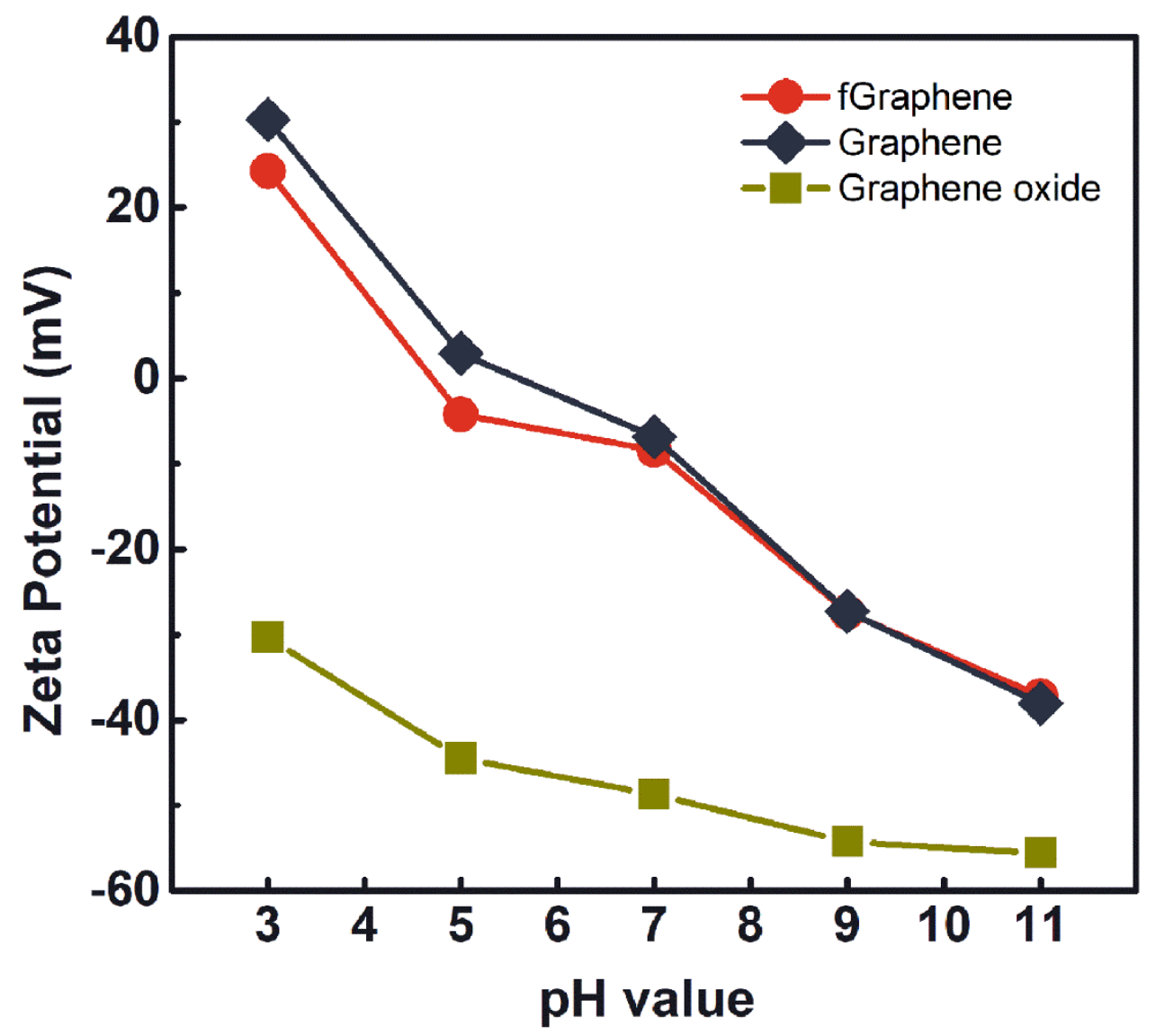

Figure S17 Zeta-potential of GO, graphene and fGraphene samples in the pH value range 3-11. 


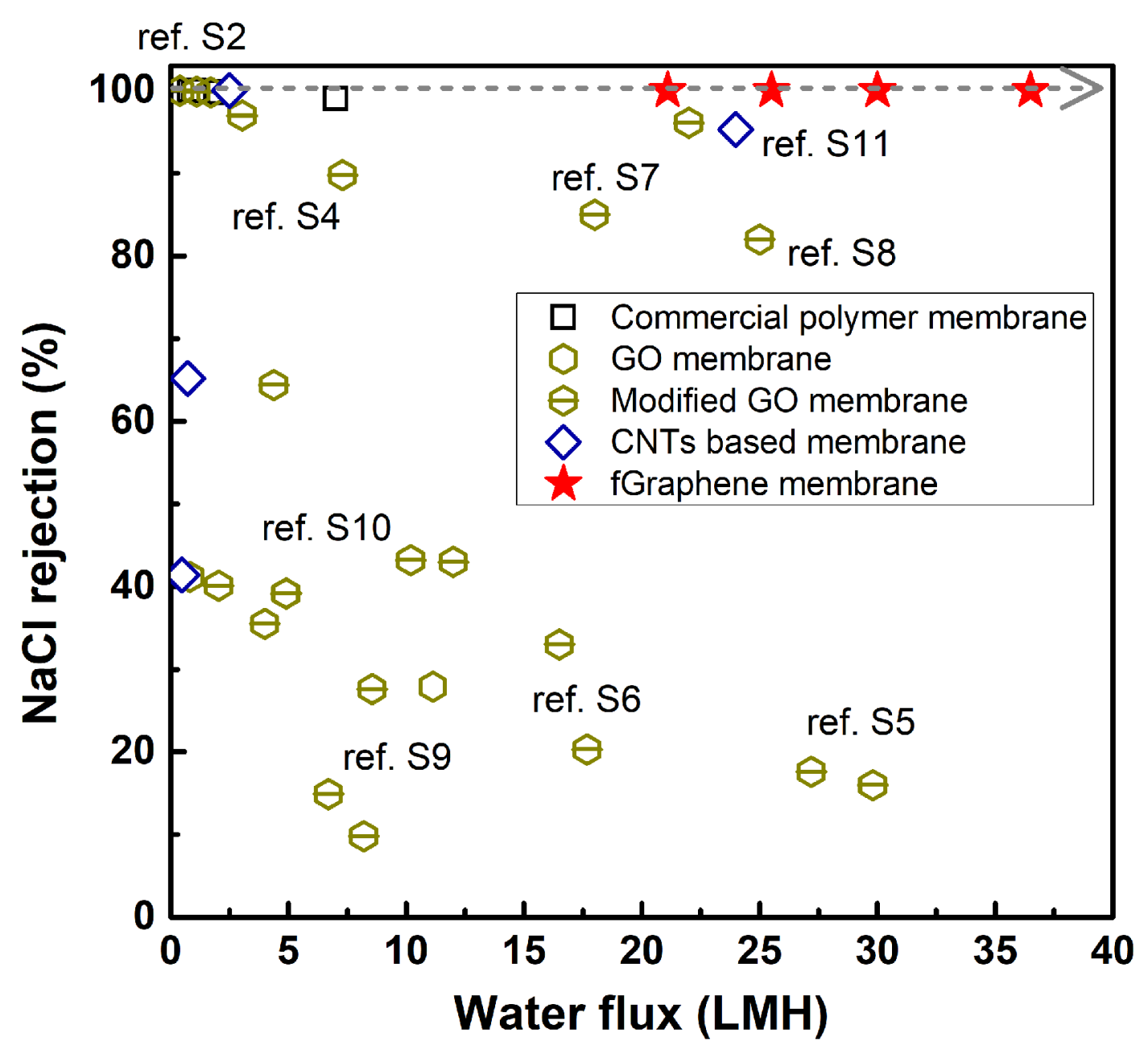

Figure S18 Comparison of the desalination performance over fGraphene membranes to those of common commercial polymer membranes, GO-based membranes and CNTs-based membranes. ${ }^{3-11}$ 


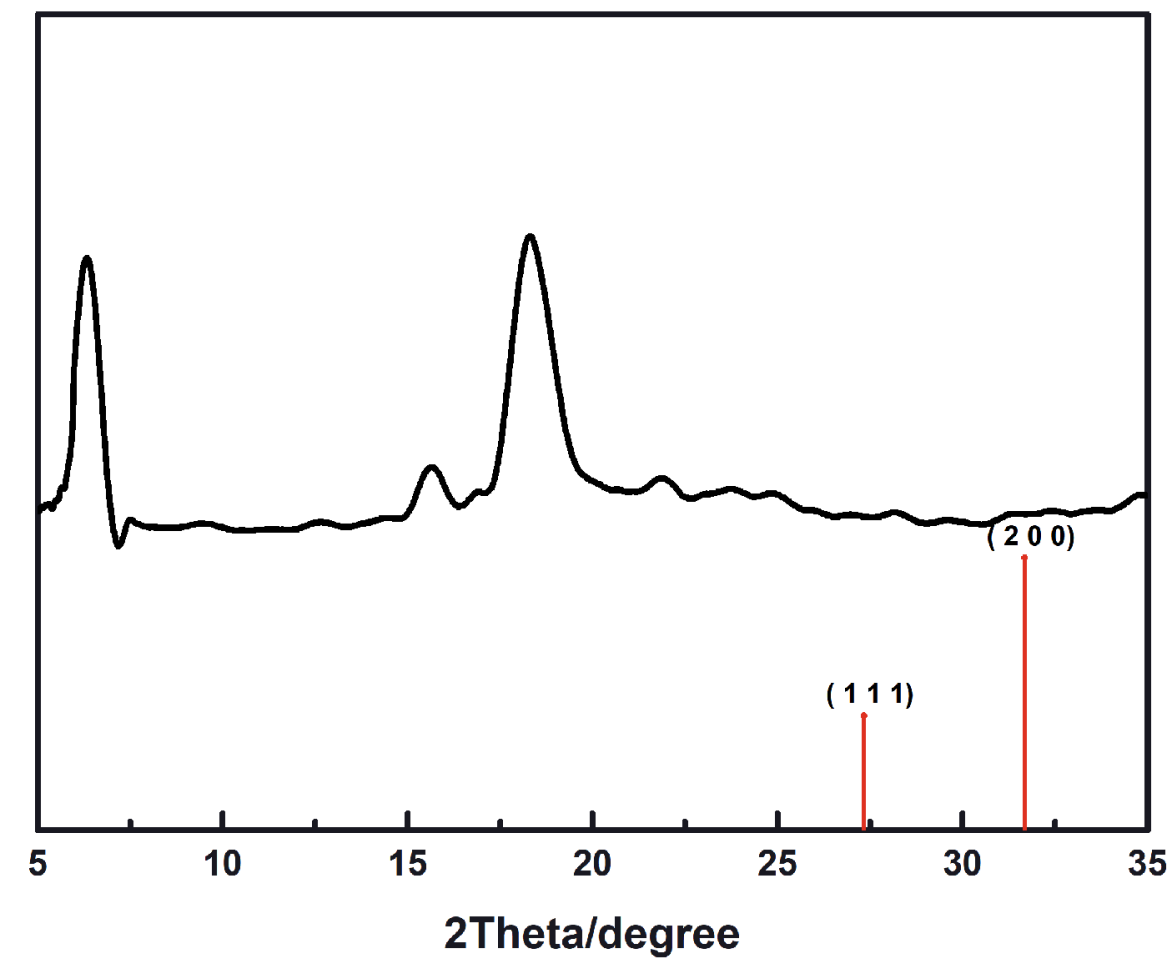

Figure S19 XRD pattern of used fGraphene membrane in comparison with the reference of $\mathrm{NaCl}$. 


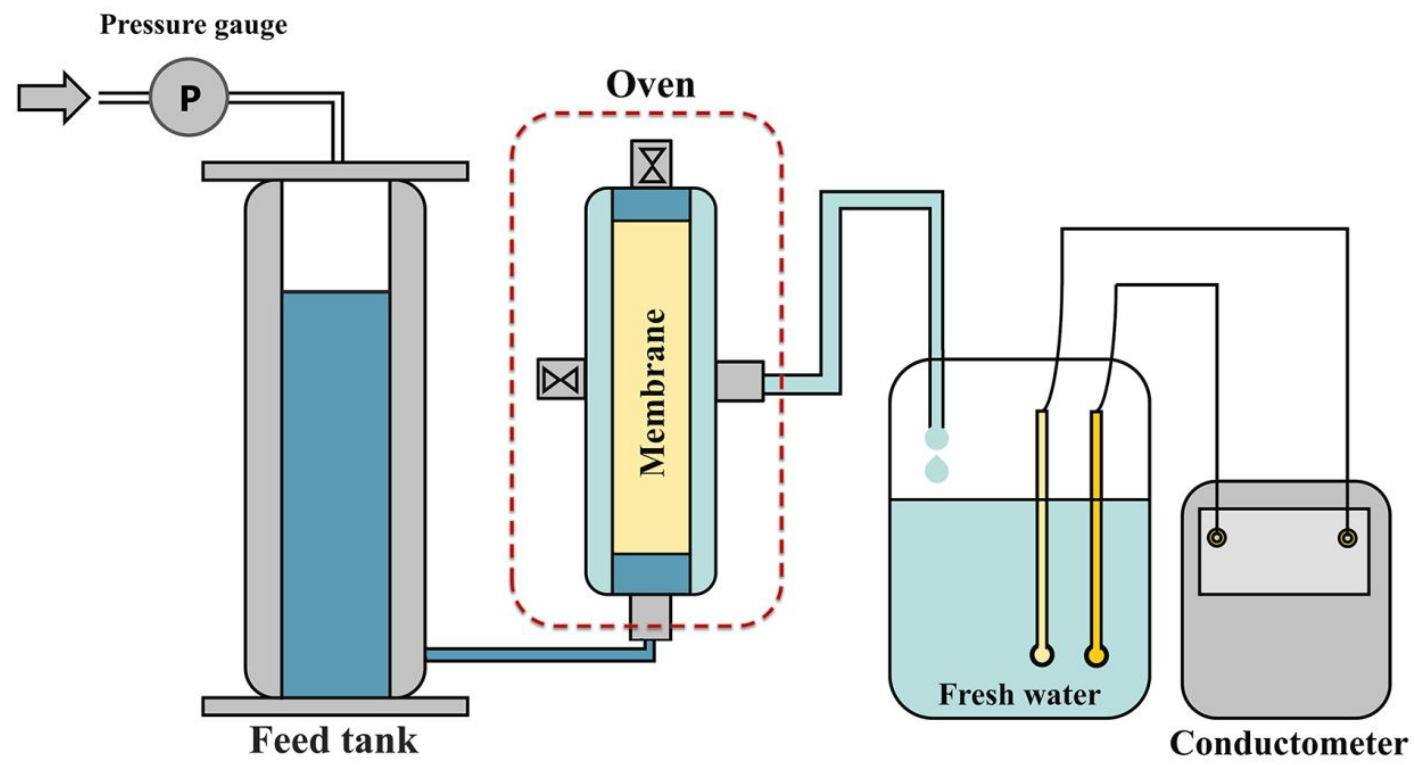

Figure S20 Schematic of the reverse osmosis process with controllable temperature. 


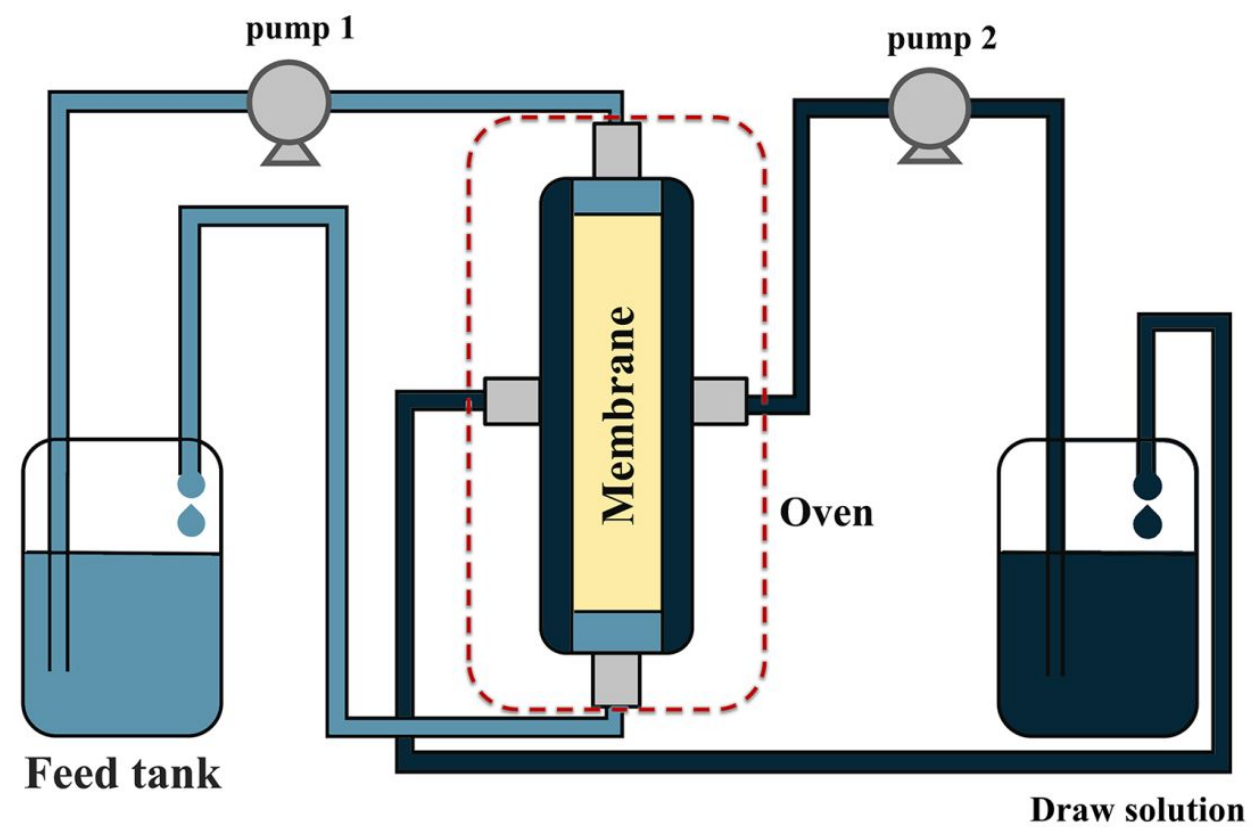

Figure S21 Schematic of the forward osmosis process with controllable temperature. 


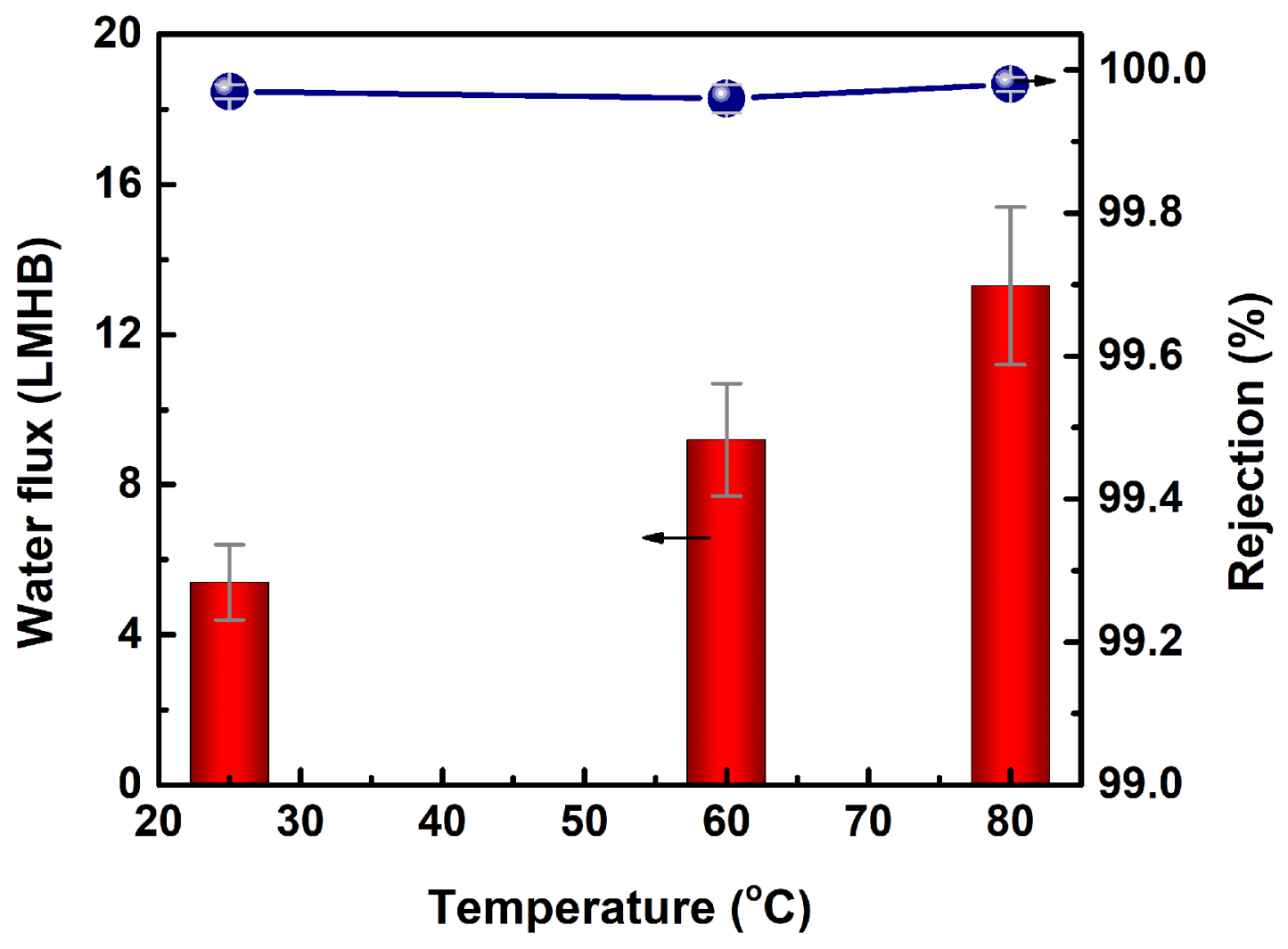

Figure S22 The desalination performance of fGraphene membrane via the reverse osmosis method (2000 ppm NaCl solution, static pressure for feeding side 3 bar). 


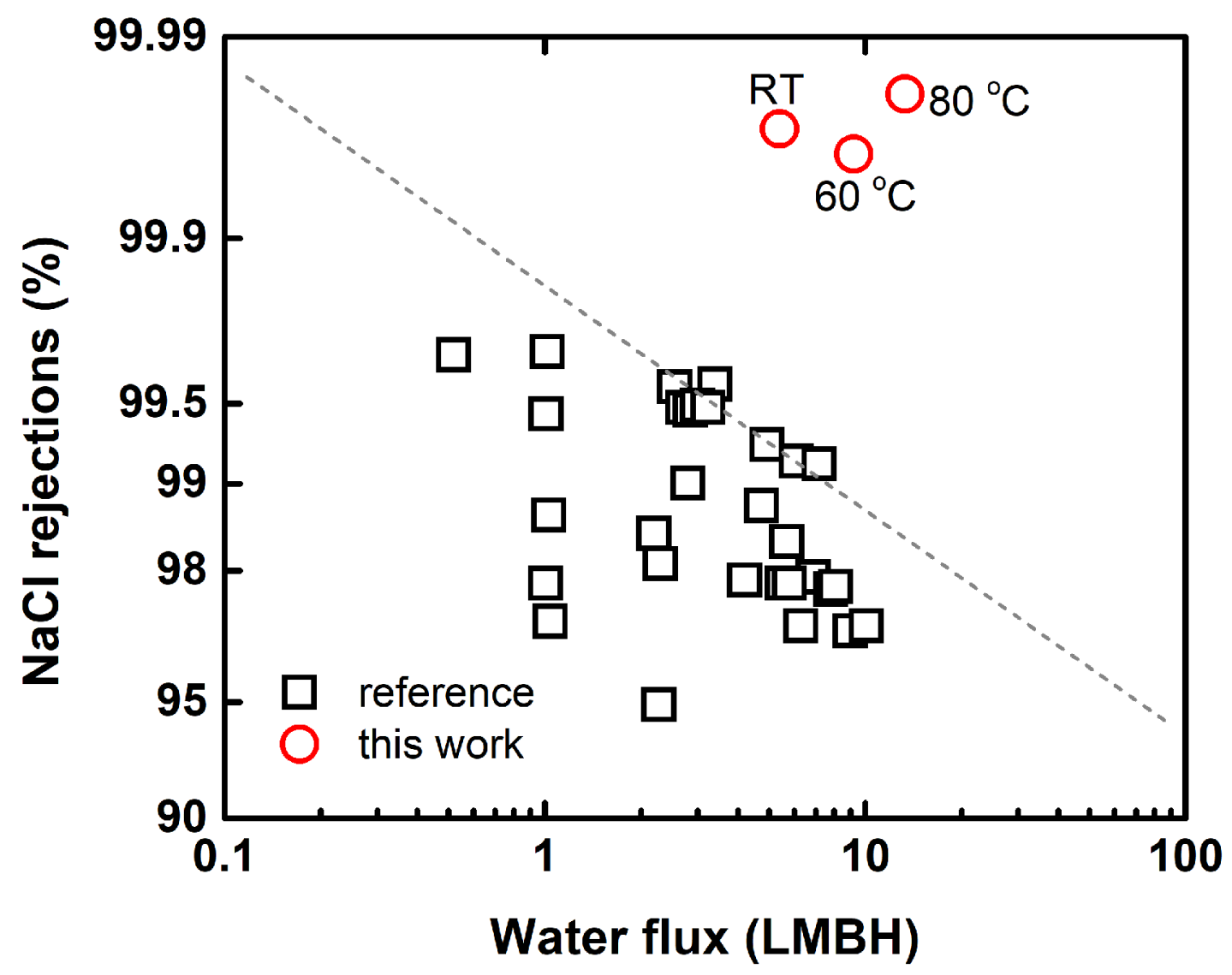

Figure S23 Comparison of RO performance of fGraphene membrane with the RO results of brackish water $(\mathrm{NaCl}$ feed solution $500-2000 \mathrm{ppm}$ ) desalination over the commercial membranes (produced by Nitto Denko Hydranautics, Dow FilmTec, and GE Osmonics) at $25^{\circ} \mathrm{C} .{ }^{12}$ 


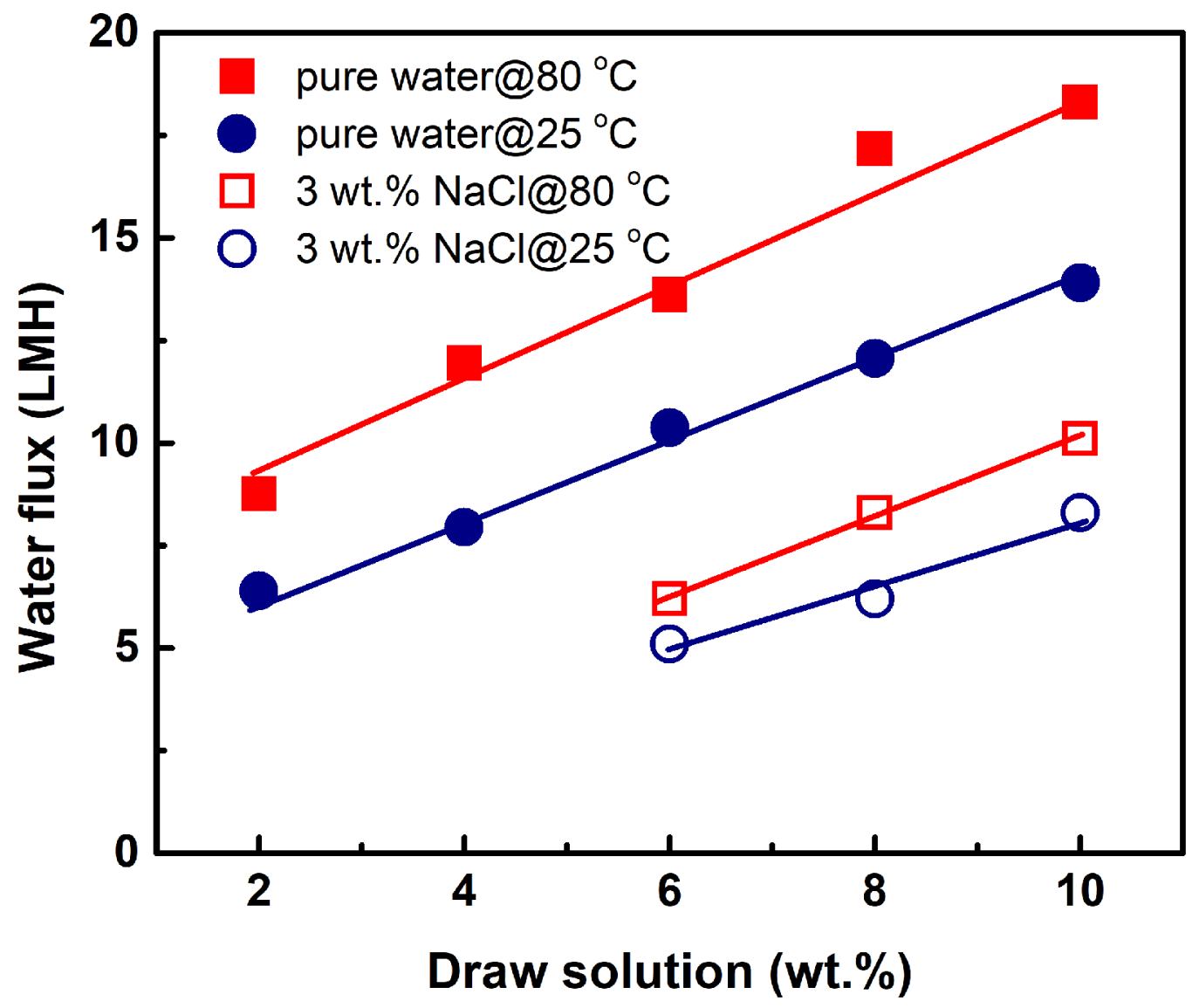

Figure S24 Water fluxes of fGraphene membrane under forward osmosis method at room temperature and $80^{\circ} \mathrm{C}$. 


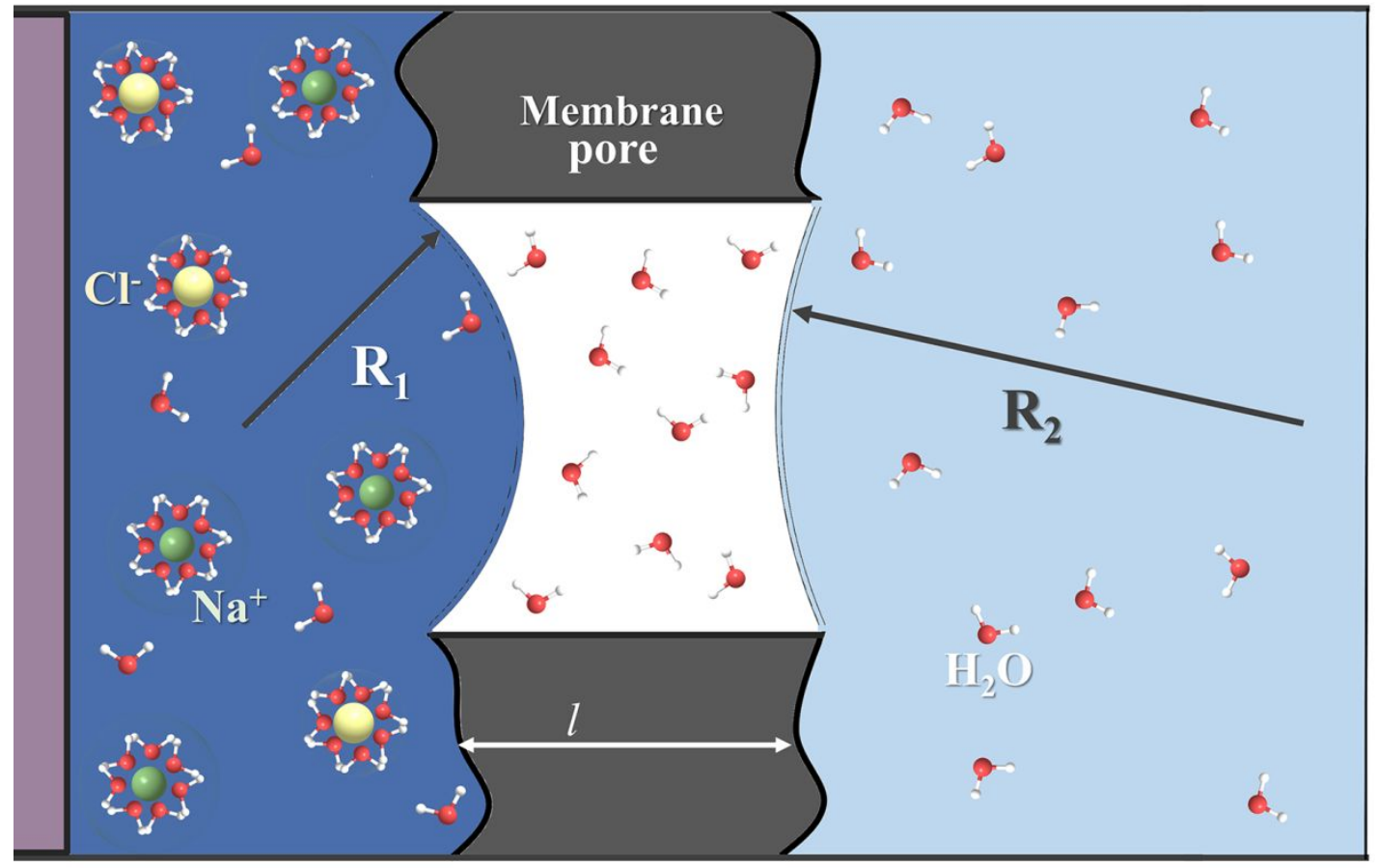

Figure S25 Schematic of a hydrophobic pore with liquid-vapor interfaces. 


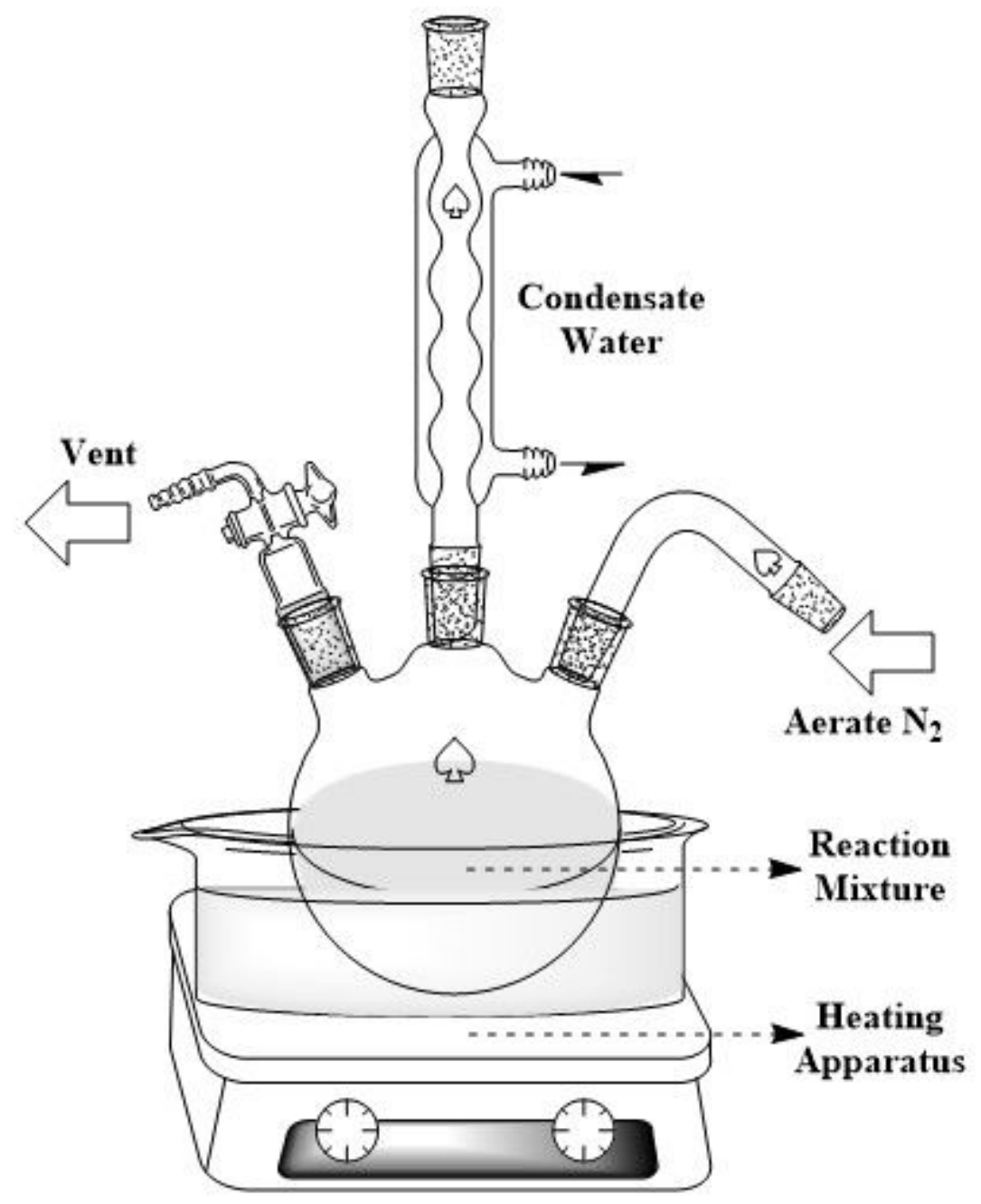

Figure S26 Schematic of thiol-ene click reaction.

Supplementary reference

1. Smolders, K.; Franken, A. C. M., Terminology for Membrane Distillation. Desalination 1989, 72, 249-262.

2. Yang, J.; Gong, D.; Li, G.; Zeng, G.; Wang, Q.; Zhang, Y.; Liu, G.; Wu, P.; Vovk, E.; Peng, Z.; Zhou, X.; Yang, Y.; Liu, Z.; Sun, Y., Self-Assembly of Thiourea-Crosslinked Graphene Oxide Framework Membranes toward Separation of Small Molecules. Adv. Mater. 2018, 30, e1705775. 3. Yang, G.; Xie, Z.; Cran, M.; Ng, D.; Easton, C. D.; Ding, M.; Xu, H.; Gray, S., Functionalizing Graphene Oxide Framework Membranes with Sulfonic Acid Groups for Superior Aqueous Mixture Separation. J. Mater. Chem. A 2019, 7, 19682-19690.

4. Wang, Q.; Li, N.; Bolto, B.; Hoang, M.; Xie, Z., Desalination by Pervaporation: A Review. Desalination 2016, 387, 46-60.

5. Han, J. L.; Haider, M. R.; Liu, M. J.; Wang, H. C.; Jiang, W. L.; Ding, Y. C.; Hou, Y. N.; Cheng, H. Y.; Xia, X.; Wang, A. J., Borate Inorganic Cross-Linked Durable Graphene Oxide Membrane Preparation and Membrane Fouling Control. Environ. Sci. Technol. 2019, 53, 1501-1508.

6. Jin, L.; Wang, Z.; Zheng, S.; Mi, B., Polyamide-Crosslinked Graphene Oxide Membrane for Forward Osmosis. J. Membrane Sci. 2018, 545, 11-18. 
7. Yu, W.; Yu, T.; Graham, N., Development of a Stable Cation Modified Graphene Oxide Membrane for Water Treatment. 2D Mater. 2017, 4, 045006.

8. Liu, T.; Yang, B.; Graham, N.; Yu, W.; Sun, K., Trivalent Metal Cation Cross-Linked Graphene Oxide Membranes for NOM Removal in Water Treatment. J. Membrane Sci. 2017, 542, 31-40.

9. Ghaffar, A.; Zhang, L. N.; Zhu, X. Y.; Chen, B. L., Scalable Graphene Oxide Membranes with Tunable Water Channels and Stability for Ion Rejection. Environ. Sci-Nano 2019, 6, 904-915.

10. Li, W.; Wu, W.; Li, Z., Controlling Interlayer Spacing of Graphene Oxide Membranes by External Pressure Regulation. ACS Nano 2018, 12, 9309-9317.

11. Ang, E. Y. M.; Toh, W.; Yeo, J.; Lin, R. M.; Liu, Z. S.; Geethalakshmi, K. R.; Ng, T. Y., A Review on Low Dimensional Carbon Desalination and Gas Separation Membrane Designs. J. Membrane Sci. 2020, 598, 117785.

12. Geise, G. M.; Park, H. B.; Sagle, A. C.; Freeman, B. D.; McGrath, J. E., Water Permeability and Water/Salt Selectivity Tradeoff in Polymers for Desalination. J. Membrane Sci. 2011, 369, 130-138. 